\title{
Development of a Vehicle-Bridge-Soil Dynamic Interaction Model for Scour Damage Modelling
}

\author{
L. J. Prendergast, ${ }^{1}$ D. Hester, ${ }^{2}$ and K. Gavin ${ }^{1}$ \\ ${ }^{1}$ School of Civil, Structural and Environmental Engineering, University College Dublin, Newstead, Belfield, Dublin 4, Ireland \\ ${ }^{2}$ School of Planning, Architecture and Civil Engineering, Queen's University Belfast, University Road, Belfast BT7 1NN, UK \\ Correspondence should be addressed to L. J. Prendergast; luke.prendergast@ucdconnect.ie
}

Received 1 July 2015; Revised 19 August 2015; Accepted 23 August 2015

Academic Editor: Ruqiang Yan

Copyright (c) 2016 L. J. Prendergast et al. This is an open access article distributed under the Creative Commons Attribution License, which permits unrestricted use, distribution, and reproduction in any medium, provided the original work is properly cited.

\begin{abstract}
Damage detection in bridges using vibration-based methods is an area of growing research interest. Improved assessment methodologies combined with state-of-the-art sensor technology are rapidly making these approaches applicable for real-world structures. Applying these techniques to the detection and monitoring of scour around bridge foundations has remained challenging; however this area has gained attraction in recent years. Several authors have investigated a range of methods but there is still significant work required to achieve a rounded and widely applicable methodology to detect and monitor scour. This paper presents a novel Vehicle-Bridge-Soil Dynamic Interaction (VBSDI) model which can be used to simulate the effect of scour on an integral bridge. The model outputs dynamic signals which can be analysed to determine modal parameters and the variation of these parameters with respect to scour can be examined. The key novelty of this model is that it is the first numerical model for simulating scour that combines a realistic vehicle loading model with a robust foundation soil response model. This paper provides a description of the model development and explains the mathematical theory underlying the model. Finally a case study application of the model using typical bridge, soil, and vehicle properties is provided.
\end{abstract}

\section{Introduction}

\subsection{Motivation for Modelling Damage in Civil Engineering} Structures. Farrar and Worden [1] give a very useful overview of the area of structural health monitoring (SHM). They point out that the motivation for governments and private companies implementing this technology is due to the economic and potentially lifesaving impact it can have. Dimarogonas [2] points out that online damage detection/monitoring started in the early 1970s when power companies started looking at developing ways of identifying defects in rotating shafts while machinery was in use. To date this kind of condition monitoring of rotating machinery has been the most successful application of SHM and it is almost entirely non-model based [1]. Effectively these machines have quite a narrow range of operating behaviours so anomalies are relatively easy to identify. As there were many of these machines in service, over time it was thus possible to develop databases that allowed specific types of damage (e.g., chipped gear teeth or damaged bearings) to be identified from particular features of the machine's vibration signature. This concept of looking for damage-sensitive features in a response signal is central to SHM. Typically an SHM algorithm works by seeking a damage feature in a response signal or by identifying a change in some characteristic of the structure when it is damaged, for example, natural frequency, mode shapes, or damping. For rotating machinery these damage features could be identified relatively easily through experiments or by correlating monitoring data with subsequent servicing records. The first instance of applying the SHM philosophy to large scale civil engineering structures was in the 1970 s and 80 s when the oil industry began attempting to apply this technology to offshore platforms. In comparison to damage arising in rotating machines, this time the structures were large and multiple damage locations/severities were possible so the nature of the damage features was typically unknown. Therefore, it was necessary to simulate candidate damage scenarios using numerical models, in order to, for example, observe how that damage affected the frequency of the platform. The idea behind this was that if this frequency was 
subsequently observed in the field it could be correlated to a given damage scenario. In general the challenges of applying vibration-based SHM to offshore platforms are significant as factors such as variations in the mass of the structure due to changes in the mass of the storage tanks and changes in the amount of marine growth on the structure can prove problematic.

The development of SHM tools for bridge engineering faces the same challenges as the oil industry in that it is very rare that one can take a full size test piece and apply damage for the purpose of developing monitoring tools. In fact it is practically unheard of in bridge engineering, as the structures themselves are just too valuable/important to interfere with. Therefore when researchers wish to try and develop a new SHM algorithm to detect a particular type of defect in the structure, the first task is to try and understand how damage affects the response of the structure. To establish this, their options (broadly speaking) are to use a computer model [3] and/or a laboratory experiment using a scaled model of the structure [4]. Occasionally it will be possible to test at full scale when a bridge is due to be retrofitted, for example, a scoured but retrofitted bridge in Italy; see [5]. However, in many cases the first option is a computer model to undertake simulations of candidate damage scenarios. The challenge, therefore, is to develop a model that outputs realistic results. A numerical model that outputs signals that are representative of those likely to be encountered on the real structure is a very useful tool when trying to develop new SHM algorithms. This paper aims to develop just such a model, as, by including vehicle-bridge interaction and soilstructure interaction, every effort is made to make the loading (applied to the bridge) and the (soil) boundary conditions of the model as realistic as possible. It should be noted that the aim of this paper is not to develop a new SHM technique for scour detection but to present the basis of a model to make it easy for others to test emerging SHM techniques by allowing them to generate realistic bridge response signals occurring due to foundation scour. Section 1.2 gives some examples of numerical models developed to simulate damage in beamlike structures, where the model outputs (e.g., acceleration, velocity, or displacement signals) were subsequently used as inputs to damage detection algorithms. This section also provides a discussion on previous works focussed on modelling scour damage.

1.2. Modelling Structural Damage for SHM Algorithm Development. Over the past two decades several authors have prepared models of damaged structures with a view to developing SHM algorithms to detect this type of damage. A particular focus has been given to models which represent localised damage in a structure (e.g., cracking or section loss). Ostachowicz and Krawczak [6] describe the different methods commonly used to model structural stiffness loss due to damage. Friswell and Penny [7] give a very useful overview on crack modelling for SHM. They point out that approaches for modelling cracks in beam type structures typically fall into three categories: local stiffness reduction [8], discrete spring models [9], and complex models in two or three dimensions. They compare the three approaches and broadly speaking they conclude that, for structural health monitoring which utilises low frequency vibration, simple models of crack flexibility based on beam elements are adequate.

Other authors have developed models to simulate the response signals of damaged beam structures to moving loads and then used the signals from these models as inputs to SHM algorithms. A number of authors have simulated the structure as having a localised loss in stiffness and then calculated the response to a moving point force [10]. Hester and González [11] modelled a similar type of damage; however they modelled the moving load as a sprung vehicle to incorporate vehicle-bridge interaction effects, that is, to make the simulated response signals as realistic as possible. This is important when numerically testing the versatility of a new SHM algorithm before applying it to a real structure. Others have postulated that the occurrence of damage will affect the damping of the bridge and prepared numerical models to simulate this by analysing the vehicle acceleration signals output from the model [12]; that is, this is an indirect monitoring approach as the vehicle response passing over a damaged bridge is used to detect the damage feature.

In terms of scour modelling, several authors have investigated the effect of scour on the static and dynamic properties of bridges using numerical methods. The principle underlying these approaches is that, during scour, loss of soil contact occurs which leads to higher applied stress over the area of soil remaining in contact with the foundation. This, coupled with the nonlinear stiffness of soils, leads to lower operational system stiffness [13]. Therefore scour presence should be detectable as a change in the dynamic properties of the structure. Ju [14] developed a 3D FE model of a bridge incorporating soil-structure and fluid-structure interaction to assess the magnitude of the change in natural frequency of the bridge with increasing scour. They validated the bridge natural frequencies from the model against a fullscale field experiment and then used the numerical model to study various scour conditions and how it affected the bridge's natural frequency. They concluded that scour causes a reduction in bridge natural frequency but the magnitude of the frequency change with scour is affected by varying foundation geometry and layering in foundation soils. Chen et al. [15] developed a full FE model of a cable-stayed bridge with a pylon and a pier. They updated the model properties to obtain a match to modal data obtained from the actual structure. They then used known data about the pylon foundation condition to obtain representative soil stiffness (matching the stiffness of the actual soil). The numerical model was then used to update the scour depth around the pier until the predicted frequency data matched the observed data. In this case, the numerical model was used to ascertain the actual scour condition around the real pier and this paper serves as a successful real-life application of a vibration-based scour detection method. Klinga and Alipour [16] developed a numerical model to assess the effect of scour on various static and dynamic performance features of a bridge. They used their model to perform pushover analyses, buckling analyses, and modal analyses under extreme scour conditions to assess the effect of scour on the various bridge elements such as 
the piles and columns. They present a number of case studies of affected performance features due to scour.

Unlike authors working in the area of bridge damage via cracking and so forth, for the purpose of scour detection, many authors have developed models to specifically perform a single task only, that is, to establish the depth of scour around a foundation element or assess the change in bridge performance under scoured conditions. No authors to date have developed a model capable of rapidly modelling a variety of bridge scour scenarios with the flexibility to test emerging SHM techniques. This paper aims to develop a numerical model that can generate dynamic signals (displacement, velocity, and acceleration) from a bridge under a variety of input loading/scour scenarios in order to allow users to develop and/or test SHM algorithms. Because of the increasing popularity of integral bridges, the structure modelled in this paper is a two-span integral bridge. Details about this type of bridge are given in Section 2.1. Section 1.3 summarises the aims of the model presented in this paper.

\subsection{Generic Algorithm for Modelling Integral Bridge Scour}

1.3.1. Method. In order to develop a representative vehiclebridge interaction model for the purpose of simulating the effect of scour on the dynamic response of an integral bridge, a number of key assumptions are made. In the first instance, to aid in the rapid generation of dynamic data, the integral bridge is assumed to act as a $2 \mathrm{D}$ frame system. The reason behind this is twofold: (i) it is assumed that the dynamic movements of interest for scour on an integral bridge predominately take place in the longitudinal direction (it is acknowledged that this assumption may not hold true for other bridge types; e.g., [15] found that for a cable-stayed bridge the first horizontal flexural and second torsional mode of the pylon were the most sensitive to scour) and (ii) 3D numerical modelling is very user and computationally costly and the resulting signals of interest are not expected to vary significantly from those in a $2 \mathrm{D}$ system. For these reasons, transverse and torsional motion of the integral bridge is neglected. The benefit of a $2 \mathrm{D}$ frame system is that a variety of representative bridges can be rapidly modelled, as the user only has to specify a relatively small number of parameters (bridge element structural and geometric properties, vehicle parameters such as axle spacing and mass). For the purpose of generating signals to test emerging SHM concepts, this is deemed adequate.

Scour can be modelled around both the central pier and the left and right abutments of the bridge and is considered as the increase in effective length of the bridge pier/abutments corresponding to a decrease in bed elevation level. The stiffness of the soil can be varied to be representative of soils from loose to dense in situ conditions that are typical of the range of ground conditions encountered in riverine environments. The foundation scour model used in this paper is derived from previously validated work undertaken by the authors (see Section 1.3.2). The method for modelling the various bridge elements, namely, deck, abutments, pier, and soil, is discussed in detail in Section 2.

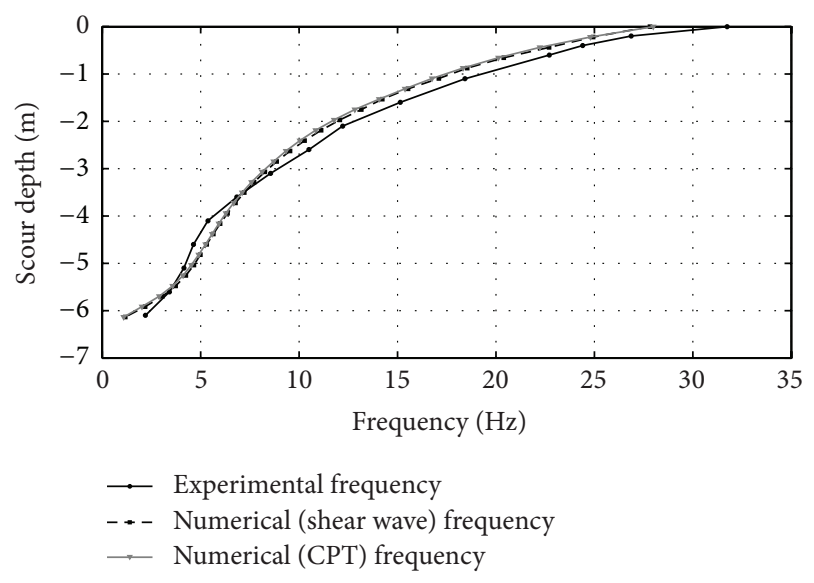

FIGURE 1: Frequency change with scour for experimental and numerical models [17].

1.3.2. Validation of Scour Model. The greatest uncertainty with regard to bridge input parameters relates to the soil stiffness. It is imperative to specify soil properties that adequately reflect the boundary stiffness effects of the soil likely to be encountered in the field for the purpose of accurate scour modelling. The method for deriving soil stiffness for scour evaluation comes from an experimental investigation undertaken by [17]. In this work, the authors measured the change in the natural frequency of a full-scale pile to manually induced scour. The field measurements were compared to numerical models developed using a Winkler spring-beam methodology (see Section 2.3). Two different methods were used to estimate the soil stiffness; the first derived soil stiffness based on the shear modulus of the soil obtained from the Multichannel Analysis of Surface Waves (MASW) (see [18]); the second method used estimates of shear modulus from a correlation to measured Cone Penetration Test (CPT) tip resistance $\left(q_{c}\right)$ data measured at the experimental site. The results of the frequency change with scour are reproduced in Figure 1.

Figure 1 shows that the experimental data and the numerical predictions from both numerical models match well. Therefore, for the purpose of scour modelling, the method developed in $[17,19]$ is used to generate representative soil profiles (and corresponding spring stiffness coefficients) for a range of soil density states. This is further discussed in the next sections.

\section{Structural Model Development}

The numerical model is developed in the MATLAB programming environment. The various components of the model are described in the following subsections. Section 2.1 describes the integral bridge to be modelled. Section 2.2 describes the mathematical modelling philosophy. Sections 2.3 and 2.4 describe how the interaction effects with foundation soil and vehicle are considered, respectively.

2.1. Integral Bridges. Integral bridges are becoming increasingly popular as they do not require a conventional expansion 


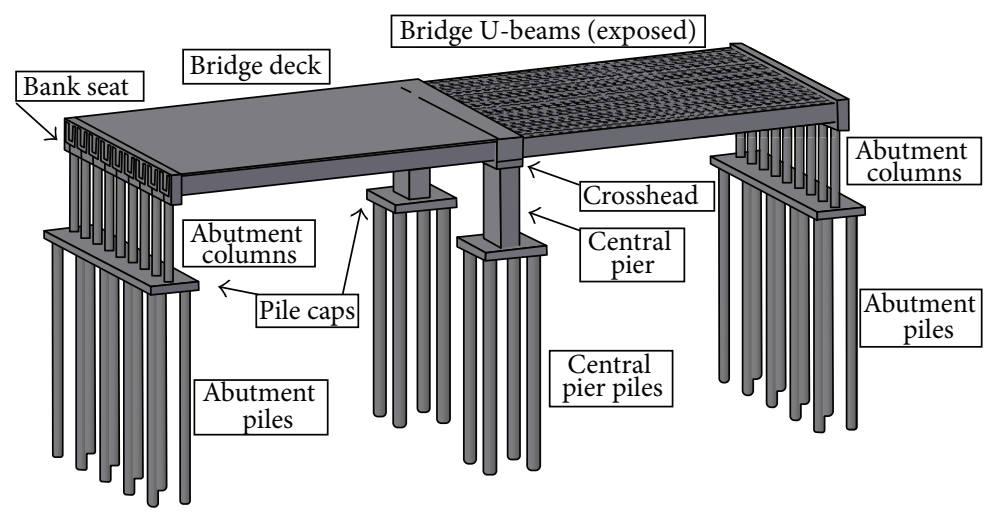

FIGURE 2: 3D rendering showing typical structural arrangement of a two-span integral bridge with flexible abutment supports.

joint. The desire to (where possible) avoid using expansion joints is due to the fact that they tend to give maintenance problems. For example, after surveying approximately two hundred concrete highway bridges in the UK, Wallbank (1989) (cited in [20]) found that expansion joints were a source of costly and disruptive maintenance work. In response to this finding, in 1996 the UK Highways Agency published Technical Note BA 42 which recommended (with some exceptions) that for new bridges up to $60 \mathrm{~m}$ in length integral construction should be considered.

The Steel Construction Institute (2015) [21] gives a useful summary on the different types of integral bridges that have been used over the years. Broadly speaking integral bridges can be split into 4 types; (i) frame abutments: this is when the abutments form a portal frame with the bridge superstructure; (ii) bank pad abutments: in this type the bank pad end support is integral to the deck and the bank pad is able to slide and rotate on the soil; (iii) flexible support abutments: this is where the bank pad is supported by piles/columns that are not in contact with the soil; that is, they have an annular space around them, and this makes them more flexible and therefore better able to absorb the thermal movements of the bridge deck; and (iv) semi-integral (screen end abutments): in this case there is an end screen wall (at the end of the deck) which is integral to the deck beams; however this wall does not provide support to the beams. Instead support is provided by some other structural element (e.g., a piled bank seat) and bearings are used to accommodate deck movements.

In this paper the flexible support abutment type is modelled, as this form of construction is most commonly used. Figure 2 shows an annotated schematic 3D rendering of a typical two-span integral bridge with flexible abutment columns. The deck is formed from prestressed concrete Ubeams and an in situ slab. The outline of the ends of the beams can be seen at the left hand end of the deck where the beams are cast into an end diaphragm/bank seat that is supported on the flexible columns. The deck beams can be seen on the right hand span (the deck slab is removed for illustrative purposes). No dimensions are indicated on the figure as the numerical model that is developed is intended to model bridges of this type but span length, pile length, section modulus, and so forth can be defined by the user. In this model the longitudinal stability of the bridge is provided primarily by the central pier which is significantly stiffer (longitudinally) than the abutment columns. Figure 3 shows an integral bridge (flexible abutment type) in its completed state. For aesthetic reasons the abutment columns are often hidden from view in the completed structure, typically using reinforced earth, which is the approach used on the bridge shown in Figure 3.

2.2. Bridge Elements. The bridge structure is modelled as a 2D frame, whereby grouped geometric properties are used to model the various structural elements, namely, the deck, abutments, central pier, and the foundation piles. The bridge elements are modelled using 6-degree-of-freedom (6-DOF) Euler-Bernoulli frame elements [22]. The individual bridge elements are assembled together to create global mass $\left[\mathbf{M}_{G}\right]$ and stiffness $\left[\mathbf{K}_{G}\right]$ matrices for the full structure, using the assembly procedure outlined in [22]. The dynamic response of the bridge is governed by the second-order matrix differential equation shown in

$$
\begin{aligned}
& {\left[\mathbf{M}_{G}\right]\left\{\begin{array}{c}
\ddot{\mathbf{x}}_{1}(t) \\
\ddot{\mathbf{x}}_{2}(t) \\
\vdots \\
\ddot{\mathbf{x}}_{N}(t)
\end{array}\right\}+\left[\mathbf{C}_{G}\right]\left\{\begin{array}{c}
\dot{\mathbf{x}}_{1}(t) \\
\dot{\mathbf{x}}_{2}(t) \\
\vdots \\
\dot{\mathbf{x}}_{N}(t)
\end{array}\right\}+\left[\mathbf{K}_{G}\right]\left\{\begin{array}{c}
\mathbf{x}_{1}(t) \\
\mathbf{x}_{2}(t) \\
\vdots \\
\mathbf{x}_{N}(t)
\end{array}\right\}} \\
& =\left\{\begin{array}{c}
\mathbf{F}_{1}(t) \\
\mathbf{F}_{2}(t) \\
\vdots \\
\mathbf{F}_{N}(t)
\end{array}\right\},
\end{aligned}
$$

where $\left[\mathbf{M}_{G}\right],\left[\mathbf{C}_{G}\right]$, and $\left[\mathbf{K}_{G}\right]$ are the $(N \times N)$ global mass, damping, and stiffness matrices for the model, respectively, and $N$ is the total number of degrees of freedom in the system. The vector $\{\mathbf{x}(t)\}$ describes the displacement of every degree of freedom for each time step in the analysis. Similarly the vectors $\{\dot{\mathbf{x}}(t)\}$ and $\{\ddot{\mathbf{x}}(t)\}$ describe the velocity and acceleration of every degree of freedom for each time step. The vector 
$\{\mathbf{F}(t)\}$ describes the external forces acting on each of the degrees of freedom for a given time step in the numerical model. The damping matrix $\left[\mathbf{C}_{G}\right]$ is calculated assuming a Rayleigh approach in line with the recommendations of [23]. The time-domain dynamic response of the system is obtained by solving (1) using numerical integration. In the model described in this paper, the Wilson- $\theta$ integration scheme is employed which is a special case of the linear acceleration method [24].

2.3. Winkler Soil Modelling. Dynamic soil-structure interaction covers a broad spectrum of applications from largestrain cyclic load-displacement regimes to small-strain low amplitude vibrations. Soil behaviour is highly nonlinear and in particular its stiffness changes nonlinearly with strain. The response of soil-structure systems is heavily dependent on the magnitude and the nature of external loading and a variety of methods exist that aim to accurately capture the behaviour of foundation systems. In this paper, it is assumed that the external loading from vehicles passing over the bridge will lead to very small lateral strains being imparted into the soil surrounding the piles. Therefore, it is assumed that the strains remain within the "small-strain" linear-elastic region of the soil response curve. This means the discretised soil impedances can be characterised by fixed value constants independent of the strain. The methods for modelling the contribution of the soil are discussed in the following subsections, namely, the mathematical assumptions underlying the process (Section 2.3.1) and the derivation of representative soil stiffness coefficients (Section 2.3.2).

2.3.1. Mathematical Assumptions. The bridge foundation comprises piled foundation elements embedded in soil. The soil is modelled using a Winkler framework, whereby the continuous soil layers are replaced by representative discrete, mutually independent, and closely spaced spring elements $[25,26]$. These spring elements have two translational degrees of freedom (2 DOFs) and permit one-dimensional uniaxial movement along the longitudinal axis of the spring. For the purpose of dynamic interaction modelling with the bridge structure, the soil springs are assumed to provide dynamic impedance only and inertial effects are ignored. In modelling terms, the springs have a null mass matrix. The stiffness matrix formulation $\left[\mathbf{K}_{s, i}\right]$ for these spring elements is shown in

$$
\left[\mathbf{K}_{s, i}\right]=k_{s, i}\left[\begin{array}{cc}
1 & -1 \\
-1 & 1
\end{array}\right], \quad k_{s, i} \geq 0,
$$

where $k_{s, i}$ is the stiffness coefficient of the $i$ th spring element. As discussed previously $k_{s, i}$ remains constant with strain in the spring due to the assumption of linear-elasticity with small soil displacements. These springs are added/integrated into the global stiffness matrix $\left[\mathbf{K}_{G}\right]$ (for the full bridge structure) by coupling/"attaching" one end of the spring to the pile nodes in the model, that is, the pile nodes located below the assumed ground line. The free end of each spring is restricted from motion to model the confining effect of the soil by setting the permissible displacement of each of these

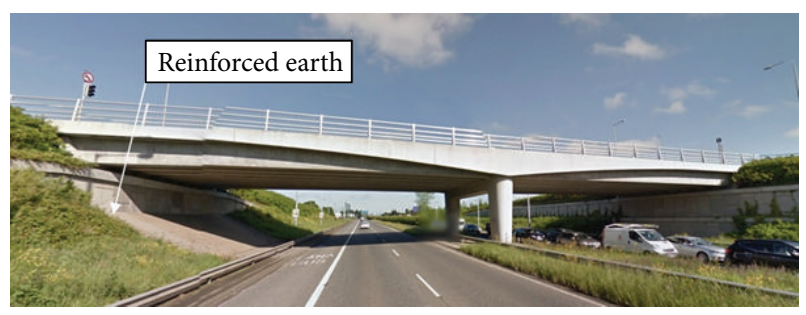

FIGURE 3: Two-span integral bridge with flexible abutment supports.

degrees of freedom to zero. Effectively every spring added to the model adds one extra degree of freedom to the global matrices $\left[\mathbf{M}_{G}\right],\left[\mathbf{C}_{G}\right]$, and $\left[\mathbf{K}_{G}\right]$. A visualisation of the spring elements is shown in Figure 5.

2.3.2. Derivation of Soil Spring Stiffness Coefficients $\left(k_{s, i}\right)$. Using a discrete spring modelling framework as per the Winkler regime, it is imperative to specify spring stiffness coefficients that accurately model the small-strain continuum soil behaviour at the soil-pile interfaces. In this paper, the method described in Prendergast et al. [19] is used to calculate representative soil stiffness coefficients for a range of soil states from loose to very dense sand.

The process involves (i) generating synthetic Cone Penetration Test (CPT) $q_{c}$ profiles that correspond to the stresses measured when a cone tip passes through a loose, medium dense, and dense sand deposit (Section 2.3.2(1)), (ii) correlating these $\mathrm{CPT} q_{c}$ profiles to profiles of the small-strain shear modulus $\left(G_{0}\right)$ for the three soil profiles adopted (Section 2.3.2(2)), and (iii) converting the shear modulus profiles into profiles of modulus of subgrade reaction $(K)$ and hence into individual spring coefficients $\left(k_{s, i}\right)$ (Section 2.3.2(3)).

(1) Hypothetical CPT $q_{c}$ Profile. As part of a standard geotechnical site investigation, Cone Penetration Tests are often carried out whereby an instrumented cone is pushed into the soil at a constant rate and the tip stresses and side sleeve friction are measured at discrete depth intervals. Lunne and Christoffersen [27] proposed an expression relating the cone tip resistance $q_{c}$ value with the soil's effective stress $\left(\sigma_{v}^{\prime}\right)$ and relative density $\left(D_{r}\right)$. A rearranged version of this expression is shown in

$$
q_{c}=60\left(\sigma_{v}^{\prime}\right)^{0.7} \exp \left(2.91 D_{r}\right) .
$$

Using (3), a hypothetical CPT $q_{c}$ profile can be derived from effective stress and relative density conditions. $D_{r}$ values of $0.3(30 \%), 0.5(50 \%)$, and $0.8(80 \%)$ are assumed to correspond to the properties of a loose, medium dense, and dense sand deposit, respectively [28], and are assumed constant with depth for uniform soils. The vertical effective stress can be calculated assuming values of bulk unit weight $\left(\gamma_{b}\right)$ of 18,19 , and $20 \mathrm{kN} \mathrm{m}^{-3}$ to approximate loose, medium dense, and dense sand deposits and the bulk unit weight of water $\left(\gamma_{w}\right)$ is assumed as $10 \mathrm{kN} \mathrm{m}^{-3}$. Although these synthetic $\mathrm{CPT} q_{c}$ profiles are idealised, they do conform quite well to the values expected for the given soil conditions [29]. 


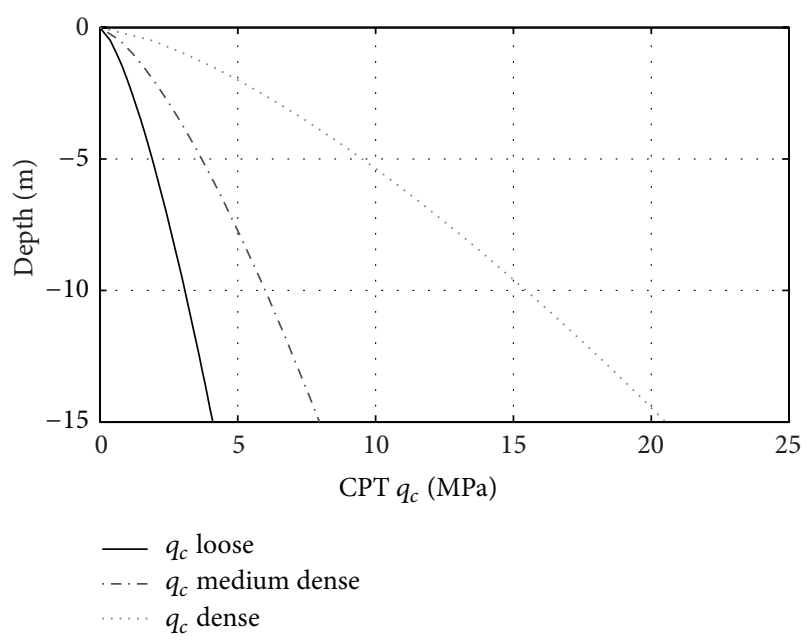

(a)

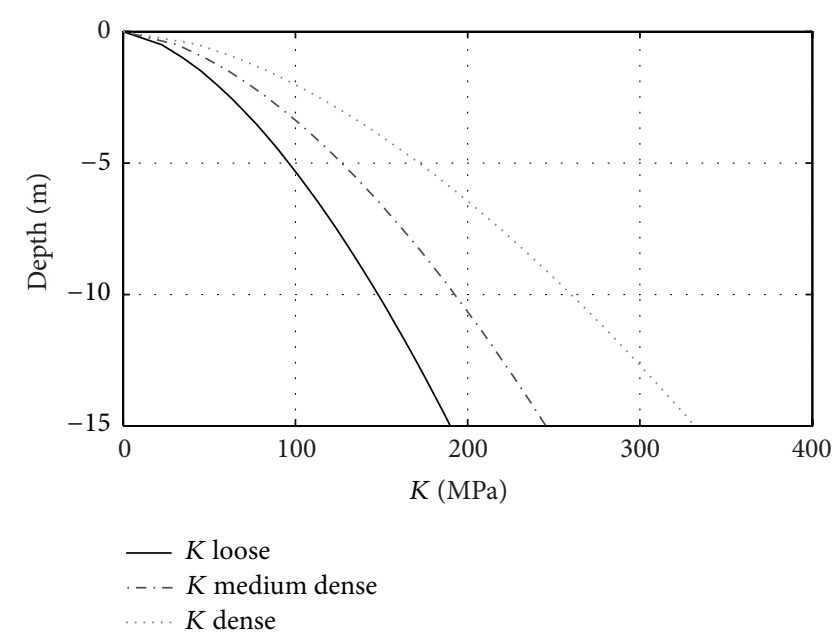

(c)

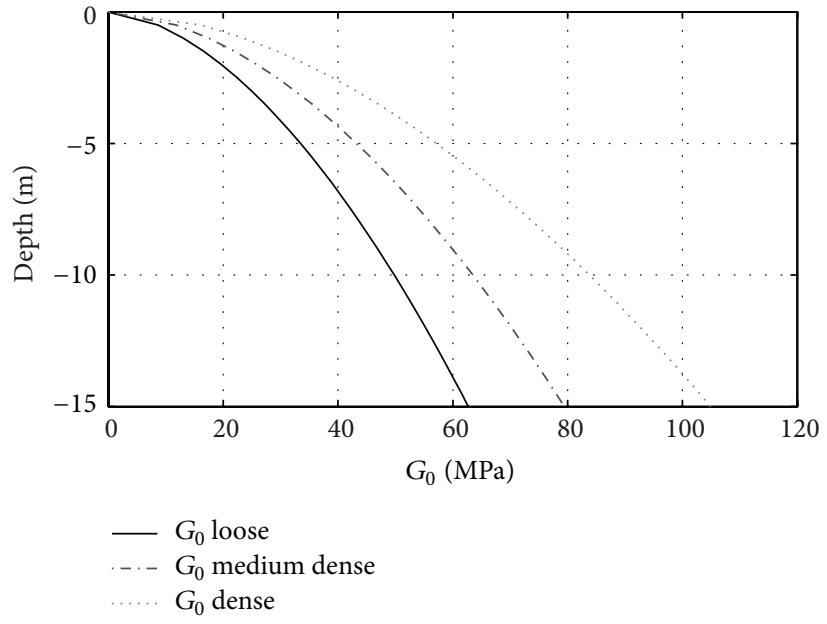

(b)

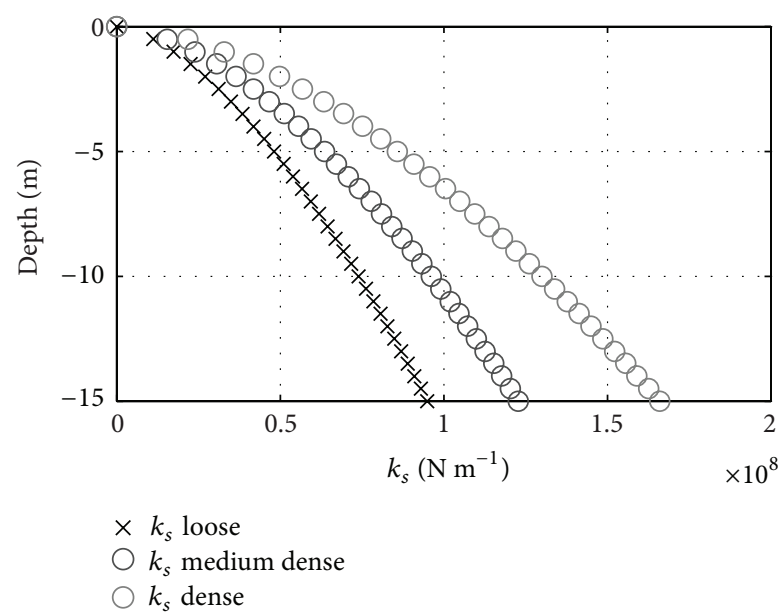

(d)

Figure 4: Example soil profiles. (a) CPT $q_{c}$ profiles for loose, medium dense, and dense sand, (b) $G_{0}$ profiles, (c) modulus of subgrade reaction $(K)$ profiles, and $(\mathrm{d})$ individual $k_{s}$ values for loose, medium dense, and dense sand.

Figure 4(a) shows typical $q_{c}$ profiles generated using this approach.

(2) Convert CPT $q_{c}$ to Profiles of $G_{0}$. There are a number of correlations linking the CPT $q_{c}$ value to the small-strain shear modulus $\left(G_{0}\right)$ for a given soil deposit. In this paper, the expression suggested in the Imperial College design method (IC-05) for driven piles in sand and clays [30] is used. This expression was originally suggested by Baldi et al. [31] and is shown in

$$
G_{0}=q_{c}\left[A+B \eta-C \eta^{2}\right]^{-1},
$$

where $A=0.0203, B=0.00125, C=1.216 E-6$, and $\eta=q_{c}\left(P_{a} \sigma_{v}^{\prime}\right)^{-0.5}$, with $P_{a}=100 \mathrm{kPa}$ and $\sigma_{v}^{\prime}=$ vertical effective stress $(\mathrm{kPa})$. Using (4), the synthetic $q_{c}$ profiles for loose, medium dense, and dense sand may be converted to profiles of the small-strain shear modulus. An example of this for a $15 \mathrm{~m}$ deep stratum is shown in Figure 4(b).

(3) Calculate Modulus of Subgrade Reaction (K) Profile. The first step in calculating the modulus of subgrade reaction from $G_{0}$ is to convert $G_{0}$ data to small-strain Young's modulus $\left(E_{0}\right)$ data using the relation shown in

$$
E_{0}=2 G_{0}(1+v),
$$

where $v$, Poisson's ratio, is assumed as 0.1 . Using $E_{0}$ profile for the soil, the modulus of subgrade reaction $(K)$ for the soilpile interface may be calculated using the expression shown in $[17,32]$

$$
K=\frac{1.0 E_{0}}{1-v^{2}}\left[\frac{E_{0} D^{4}}{E_{p} I_{p}}\right]^{1 / 12},
$$

where $D$ is the effective diameter of the pile group (m), $E_{p}$ is Young's modulus of the pile material $\left(\mathrm{N} \mathrm{m}^{-2}\right)$, and $I_{p}$ is 


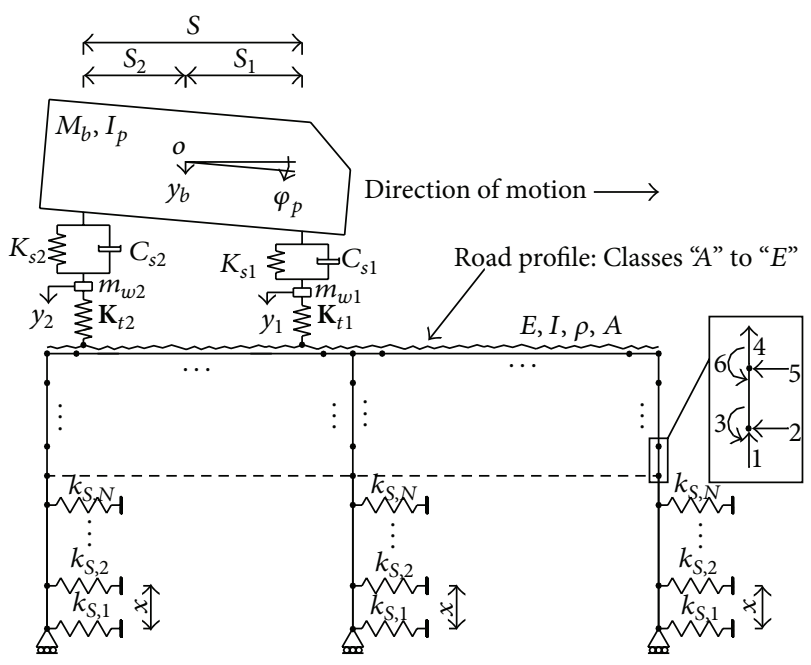

FIGURE 5: Continuum model of half car crossing an integral bridge (not to scale).

the effective moment of inertia of the pile group $\left(\mathrm{m}^{4}\right)$. The effective diameter of the pile group is obtained by summing the diameters of the individual piles in the group and the effective moment of inertia of the pile group is obtained in the same way. Individual soil stiffness coefficients $\left(k_{s, i}\right)$ are calculated by multiplying $K$ value at a given spring depth by the spacing between springs at that depth.

An example of converting $G_{0}$ data from Figure 4(b) into a profile of the modulus of subgrade reaction $(K)$ is shown in Figure 4(c) and hence that into individual spring coefficients $\left(k_{s, i}\right)$ is shown in Figure $4(\mathrm{~d})$. In this figure, the effective diameter of the pile group is taken as $6 \mathrm{~m}$ ( 8 piles $\times$ $0.75 \mathrm{~m}$ diameter each) and the pile material is assumed to be concrete.

\subsection{Vehicle Model and Interaction}

2.4.1. Overview of Vehicle-Bridge Interaction (VBI) Problem. Having developed a finite element model of bridge-soil interaction the next step toward implementing a VehicleBridge-Soil Dynamic Interaction (VBSDI) model is to model how the vehicle applies loading to the bridge as it passes over it. The important point to note is that as a vehicle passes over a bridge the loading it applies to the bridge is not constant as it is affected by the road profile (surface roughness), the speed of the vehicle, and the properties of the vehicle.

The fundamental challenge with vehicle-bridge interaction modelling is that the movement of the bridge influences movements of the vehicle, which in turn influence movements of the bridge. When studying vehicle-bridge interaction problems two sets of equations can be written: one set for the vehicle and one set for the bridge. To satisfy compatibility the contact forces in both subsystems must be the same and it is these contact forces that make the two sets of equations coupled. Yang et al. [23] give an overview of the problem as well as a description of some commonly used techniques such as the iterative method, the dynamic condensation method, and Yang's VBI element. Each of the
TABLE 1: Typical parameters for truck model.

\begin{tabular}{llc}
\hline \multicolumn{2}{c}{ Dimensional data } \\
\hline $\begin{array}{l}\text { Dimensions } \\
(\mathrm{m})\end{array}$ & Wheel base $(S)$ & 5.5 \\
& Dist. from centre of mass to front axle $\left(S_{1}\right)$ & 3.66 \\
& Dist. from centre of mass to rear axle $\left(S_{2}\right)$ & 1.84 \\
\hline \multicolumn{3}{c}{ Mass and inertia } \\
\hline Mass $(\mathrm{kg})$ & Front wheel/axle mass $\left(m_{w 1}\right)$ & 700 \\
& Rear wheel/axle mass $\left(m_{w 2}\right)$ & 1,100 \\
\hline Inertia $\left.(\mathrm{kg} \mathrm{m})^{2}\right)$ & Pitch moment of inertia of truck $\left(I_{p}\right)$ & 13,300 \\
\hline & \multicolumn{1}{c}{ Suspension } & 41,008 \\
\hline Spring stiffness & Front axle $\left(K_{s 1}\right)$ & 400 \\
$\left(\mathrm{kN} \mathrm{m}^{-1}\right)$ & Rear axle $\left(K_{s 2}\right)$ & 1,000 \\
\hline $\begin{array}{l}\text { Damping } \\
\left(\mathrm{kN} \mathrm{s} \mathrm{m}^{-1}\right)\end{array}$ & Front axle $\left(C_{s 1}\right)$ & 10 \\
\hline Tyre stiffness & Rear axle $\left(C_{s 2}\right)$ & 10 \\
$\left(\mathrm{kN} \mathrm{m}^{-1}\right)$ & Front axle $\left(\mathbf{K}_{t 1}\right)$ & 1,750 \\
\hline
\end{tabular}

methods has certain advantages and disadvantages; however, for the purposes of this study the iterative approach was suitable. Iterative techniques have been used by Green and Cebon [33] and Yang and Fonder [34] and are described in more detail in Section 2.4.4.

Before describing how vehicle-bridge interaction is implemented it is first useful to look at the bridge-vehicle model; see Figure 5. In this arrangement the vehicle has 4 degrees of freedom: the pitch of the sprung body mass $\varphi_{p}(t)$, the displacement of the sprung body mass $\mathbf{y}_{b}(t)$, the displacement of unsprung mass $1 \mathbf{y}_{1}(t)$, and the displacement of unsprung mass $2 \mathbf{y}_{2}(t)$. Unsprung masses 1 and 2 represent the masses of the front and rear axle assembly.

2.4.2. Vehicle Properties. In this study the vehicle modelled is a two-axle truck. The geometry of the test truck can be tailored by the user to represent any type of two-axle truck; that is, the sprung body mass, axle spacing, unsprung axle mass, suspension damping and stiffness, and so forth can all be modified by the user. Harris et al. [35] give the typical suspension and tyre properties for this kind of vehicle and Table 1 gives a summary of the vehicle properties that were used in this study.

2.4.3. Generating a Road Profile. The road profile is simply an array of numbers that defines the height of the road surface at discrete intervals along the length of the bridge (e.g., every $1 \mathrm{~cm}$ ). The length of road profile necessary depends on the span of the bridge, the wheel base of the vehicle, and the desired approach distance. The purpose of modelling the movement of the vehicle as it approaches the bridge is to allow it to reach a steady state of vibration before it reaches the bridge, thereby making its subsequent interaction with the bridge more realistic. A typical approach length used is of the order of $100 \mathrm{~m}$. Cebon [36] describes how the topography of a given road profile can be classified in accordance with the 
ISO standard; for example, a road profile can be classified as "very good," "good," “average," "poor," or "very poor." Cebon also describes how an artificial road surface topography of a given roughness/classification can be generated for use in time-domain vehicle vibration simulations. While the road profile generator defines the height of equally spaced points along the road (e.g., spatially distributed every $1 \mathrm{~cm}$ ), it is inappropriate to use these heights directly in the model. The reason for this is that the wheel of the truck is not supported by just one road profile ordinate. Typical truck tyres span approximately $24 \mathrm{~cm}$; therefore the wheel rests upon 24 road profile ordinates simultaneously (for ordinates spaced at $1 \mathrm{~cm}$ intervals). To take account of this fact the road profile used in the model is obtained by applying a moving average filter to the road profile given by the road profile generator to obtain the average ordinate height over the span of the wheel. Finally it is necessary to extract from the global road profile the road profile experienced by the front axle on the approach section $r_{1 A}(t)$ and on the bridge section $r_{1}(t)$ and to do the same for the rear axle. (The road profile is generated spatially; however for simulation purposes the important thing to know is the height of the road profile ordinate under each axle at each time step.) It is also important to note that the purpose behind incorporating a road profile in the model is to afford a more realistic treatment of the vehicle-bridge interaction problem and to allow researchers to test the resilience of emerging SHM techniques under a variety of conditions. Road profiles are generated randomly according to the specified road profile classification and it is not intended to model the road profile of a specific bridge. Figure 6 shows example road profiles generated for a $50 \mathrm{~m}$ long bridge span. The road surface roughness is varied from Class " $A$ " (very good) to Class " $E$ " (very poor) for the purpose of illustration.

2.4.4. Iterative VBI Procedure. By using the equations of Fryba [37] it is possible to develop a stiffness $\left[\mathbf{K}_{v}\right]$, mass $\left[\mathbf{M}_{v}\right]$, and damping $\left[\mathbf{C}_{v}\right]$ matrix for the vehicle. The dynamic response of the vehicle is modelled as shown in

$$
\begin{aligned}
& {\left[\mathbf{M}_{v}\right]\left\{\begin{array}{c}
\ddot{\varphi}_{p}(t) \\
\ddot{\mathbf{y}}_{b}(t) \\
\ddot{\mathbf{y}}_{1}(t) \\
\ddot{\mathbf{y}}_{2}(t)
\end{array}\right\}+\left[\mathbf{C}_{v}\right]\left\{\begin{array}{c}
\dot{\varphi}_{p}(t) \\
\dot{\mathbf{y}}_{b}(t) \\
\dot{\mathbf{y}}_{1}(t) \\
\dot{\mathbf{y}}_{2}(t)
\end{array}\right\}+\left[\mathbf{K}_{v}\right]\left\{\begin{array}{c}
\varphi_{p}(t) \\
\mathbf{y}_{b}(t) \\
\mathbf{y}_{1}(t) \\
\mathbf{y}_{2}(t)
\end{array}\right\}} \\
& =\left\{\mathbf{F}_{v}\right\},
\end{aligned}
$$

where $\mathbf{F}_{v}$ is the vector of forces acting on the vehicle degrees of freedom for a given time step. In the first instance, the vehicle is run across the bridge assuming the bridge does not experience any deflection. The displacement of each vehicle axle, $\mathbf{y}_{1}(t)$ and $\mathbf{y}_{2}(t)$, is then obtained using the Wilson- $\theta$ method applied to (7) and the contact forces between the vehicle and the road surface for each time step are calculated in

$$
\left\{\begin{array}{l}
\mathbf{F}_{1}(t) \\
\mathbf{F}_{2}(t)
\end{array}\right\}=\left[\begin{array}{cc}
\mathbf{K}_{t 1} & \mathbf{0} \\
\mathbf{0} & \mathbf{K}_{t 2}
\end{array}\right]\left\{\begin{array}{l}
\mathbf{y}_{1}(t) \\
\mathbf{y}_{2}(t)
\end{array}\right\},
$$

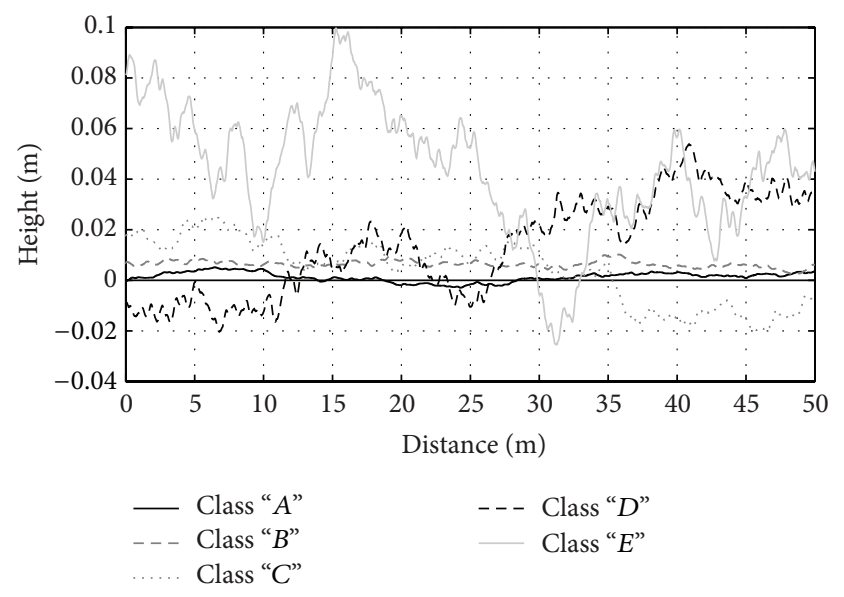

Figure 6: Example road profiles: Class " $A$ " to Class " $E$ ” for a $50 \mathrm{~m}$ long bridge.

where $\mathbf{K}_{t 1}$ and $\mathbf{K}_{t 2}$ are the front and rear tyre stiffness, respectively. The contact forces obtained in (8) can then be applied to the FE model of the bridge. This is achieved by defining $\mathbf{F}(t)$ in (1) using Hermitian shape functions to apportion the axle loads $\mathbf{F}_{1}(t)$ and $\mathbf{F}_{2}(t)$ to the bridge nodes since the position of the loads $\mathbf{x}(t)$ at every time step is known. Once $\mathbf{F}(t)$ has been populated the Wilson$\theta$ method is used to calculate the displacement of each of the bridge degrees of freedom at each time step. Since the first run of the vehicle assumed no bridge displacement, the process must be iterated to take account of the bridge displacement changing the height of the road profile experienced by each axle at each time step. The latest road profile affects the contact forces, which in turn affect the bridge displacement, which affects the road profile and so forth. The road profile is continually updated by subtracting the bridge displacements until convergence is achieved. Guidance on convergence for simulations of this type is given in [33]. Convergence in this study is deemed to have occurred when the maximum difference in displacement (for all degrees of freedom) between the current time step and the previous time step is less than $1 \%$ of the max displacement of the bridge; see

$$
\left|\frac{\Delta_{i}-\Delta_{i-1}}{\Delta_{\max }}\right| \leq 0.01
$$

\section{Modelling Algorithms}

3.1. Scour Modelling. In this section, the output of signals of interest from the system and the postprocessing required to make them "analysis ready" is discussed. As mentioned in previous sections, the model is capable of outputting dynamic displacement, velocity, and acceleration signals from a multitude of points on the bridge structure. Moreover, the model is also capable of outputting dynamic displacement, velocity, and acceleration signals from the vehicle model as it traverses the bridge. Using the signals arising on the bridge structure itself allows the user to test direct SHM techniques, that is, methods that rely on sensors being placed on the actual 


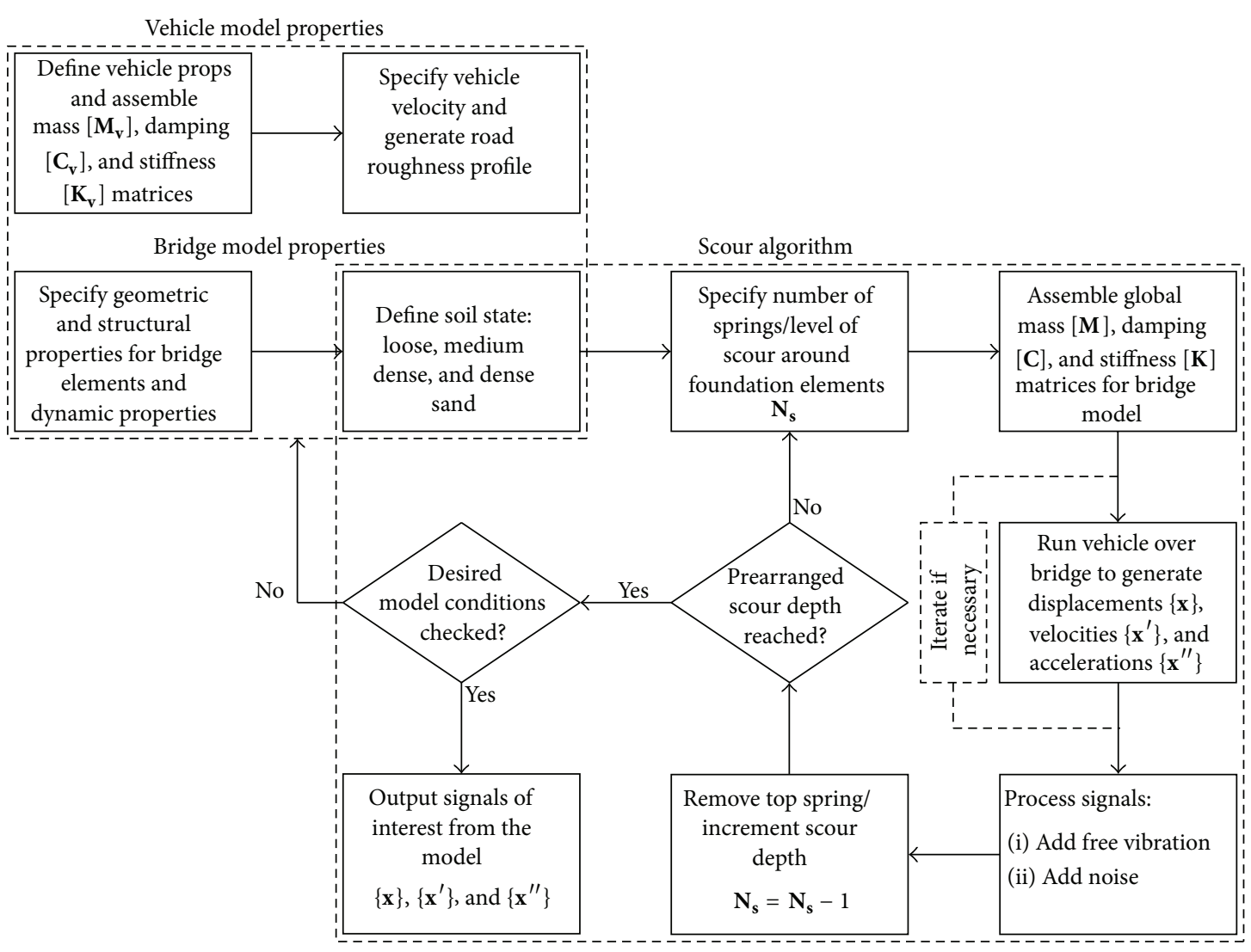

FIGURE 7: Scour modelling algorithm to generate analysis signals.

structure to detect damage features. Using the signals arising on the vehicle system allows the user to test indirect methods of damage detection, which use sensors placed on the vehicle (i.e., axles, body) to detect damage on the bridge as it traverses. The generic algorithm to generate signals of interest from the bridge is shown in the flow diagram in Figure 7. In order to create usable signals for analysis, it is often necessary to simulate damped free vibration of the bridge after the vehicle has departed the structure (this is because high vehicle speeds can result in very short signals which is often problematic from a signal processing point of view). The model also has the facility to add "measurement noise" that would be present in real signals. This is discussed in Section 3.2. Scour is modelled as the removal of springs from around the foundation element of interest (pier, abutments) starting with the spring nearest the top.

3.2. Adding Noise to Numerical Signals. Signals recorded on a real structure will contain measurement noise; therefore for the purpose of a fair assessment of a given SHM algorithm, the presence of noise in the numerically generated signals should be accounted for. The model developed in this paper allows for two different methods to add noise to the "clean" numerical signals. The first method is based on Zhu and Law [10] and the second method is based on Lyons [38]. The Zhu and Law approach allows the user to specify a percentage noise level to be added to the signal of interest and is formulated as follows:

$$
\operatorname{sig}_{\text {NOISE }}=\operatorname{sig}_{\text {CLEAN }}+E_{p} N_{\text {NOISE }} \sigma\left(\operatorname{sig}_{\text {CLEAN }}\right),
$$

where $\operatorname{sig}_{\text {NOISE }}$ is the noisy (corrupted) signal, $E_{p}$ is the percentage of added noise, $N_{\text {NOISE }}$ is a standard normal distribution vector with zero mean value and unit standard

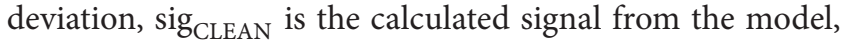
and $\sigma\left(\operatorname{sig}_{\text {CLEAN }}\right)$ is the standard deviation. Specifying $E_{p}$ values of 0.01 to 0.05 adds $1 \%$ to $5 \%$ of noise, respectively, to the clean signal, for example.

The second method used to add noise is based on the signal-to-noise ratio (SNR), given in [38]

$$
\mathrm{SNR}=10 \log _{10} \frac{\text { Signal Power }}{\text { Noise Power }},
$$

where SNR is the ratio of the strength of a signal carrying information equating to that of unwanted interference. This equation can be rearranged to give

$$
\sigma_{N}=\sqrt{\text { Noise Power }}=\sqrt{\frac{\text { Signal Power }}{\exp \left(\left(\operatorname{SNR} \cdot \log _{e}(10)\right) / 10\right)}},
$$

where $\sigma_{N}$ is the variance of the noise. Using (12), noise signals with different signal-to-noise ratios can be added to 
TABLE 2: Case study bridge properties.

\begin{tabular}{|c|c|c|c|}
\hline \multicolumn{4}{|c|}{ Bridge deck } \\
\hline Young's modulus $(E)(\mathrm{MPa})$ & 35000 & $\mathrm{X}$-sectional area $\left(\mathrm{m}^{2}\right)$ & 9.516 \\
\hline Moment of inertia $\left(\mathrm{m}^{4}\right)$ & 2.9487 & Concrete density $\left(\mathrm{kg} \mathrm{m}^{-3}\right)$ & 2400 \\
\hline Spans & 2 & Span length $(\mathrm{m})$ & 25 \\
\hline \multicolumn{4}{|c|}{ Bridge abutment columns } \\
\hline Young's modulus $(E)(\mathrm{MPa})$ & 35000 & Total X-sectional area $\left(\mathrm{m}^{2}\right)$ & 1.7671 \\
\hline Total moment of inertia $\left(\mathrm{m}^{4}\right)$ & 0.0276 & Concrete density $\left(\mathrm{kg} \mathrm{m}^{-3}\right)$ & 2400 \\
\hline Number of columns & 9 & Column length $(\mathrm{m})$ & 6 \\
\hline \multicolumn{4}{|c|}{ Bridge pier } \\
\hline Young's modulus $(E)(\mathrm{MPa})$ & 35000 & Total X-sectional area $\left(\mathrm{m}^{2}\right)$ & 7.22 \\
\hline Total moment of inertia $\left(\mathrm{m}^{4}\right)$ & 1.137 & Concrete density $\left(\mathrm{kg} \mathrm{m}^{-3}\right)$ & 2400 \\
\hline Number of columns & 2 & Column length $(\mathrm{m})$ & 6 \\
\hline \multicolumn{4}{|c|}{ Abutment piles } \\
\hline Young's modulus $(E)(\mathrm{MPa})$ & 35000 & Total X-sectional area $\left(\mathrm{m}^{2}\right)$ & 2.827 \\
\hline Total moment of inertia $\left(\mathrm{m}^{4}\right)$ & 0.0636 & Concrete density $\left(\mathrm{kg} \mathrm{m}^{-3}\right)$ & 2400 \\
\hline Number of piles & 10 & Pile length (m) & 15 \\
\hline \multicolumn{4}{|c|}{ Pier piles } \\
\hline Young's modulus $(E)(\mathrm{MPa})$ & 35000 & Total X-sectional area $\left(\mathrm{m}^{2}\right)$ & 3.534 \\
\hline Total moment of inertia $\left(\mathrm{m}^{4}\right)$ & 0.1243 & Concrete density $\left(\mathrm{kg} \mathrm{m}^{-3}\right)$ & 2400 \\
\hline Number of piles & 8 & Pile length (m) & 15 \\
\hline
\end{tabular}

the original "clean" signal obtained directly from the numerical model. This process is shown in

$$
\operatorname{Sig}_{\text {NOISE }}=\sigma_{N}[\text { rand }]+\operatorname{Sig}_{\text {CLEAN }} \text {. }
$$

The addition of noise to the signals allows for a more robust testing of a given SHM scheme as, in general, measurement noise can inhibit the accuracy of a given SHM scheme.

\section{Example Signals from Developed Model}

In this section, some example signals simulated by the model and their response features are shown. Section 4.1 displays some typical bridge signals and Section 4.2 displays some vehicle signals calculated when the vehicle is crossing the bridge. The case study bridge modelled is a two-span integral bridge with flexible abutments; each span is $25 \mathrm{~m}$ in length (Figure 2 shows a 3D schematic of the bridge). The bridge deck is formed using nine U10 bridge beams [39] supporting a $200 \mathrm{~mm}$ thick deck slab. The bridge abutments are formulated with nine concrete columns supporting the bridge beams, each column $500 \mathrm{~mm}$ in diameter, and spaced at $1900 \mathrm{~mm}$ centres. Two stiff piers support the deck at the centre and have plan dimensions of $1375 \mathrm{~mm} \times 2625 \mathrm{~mm}$. The abutment columns rest on a pile cap under which ten, $15 \mathrm{~m}$ long $(0.6 \mathrm{~m}$ diameter) bored concrete piles are placed. A loose sand soil profile is generated as per the analysis in Section 2.3.2. For the purpose of modelling, scour around an individual foundation element is assumed to be uniform along the transverse length of a given support. As mentioned previously, the model is idealised as a $2 \mathrm{D}$ frame with group properties adopted for the various structural members. The structural properties shown in Table 2 are used for the simulations presented in this section.
4.1. Bridge Signals. Some typical response signals from the bridge due to the vehicle crossing are shown in Figure 8. For this analysis, the vehicle (properties shown in Table 1) traverses the bridge at $50 \mathrm{~km} \mathrm{hr}^{-1}$ over a Class " $A$ " road surface (shown in Figure 6) and a zero scour condition is assumed. Ten seconds of free vibration is also calculated and the analysis assumes damped vibration at $2 \%$. The three plots on the left side of the figure (i.e., (a), (c), and (e)) show the vertical displacement, velocity, and acceleration of the midpoint of the left span ( span 1) of the bridge resulting from the passage of the vehicle across the bridge. The displacement response in part (a) of the figure is dominated by the static bridge response. When the truck is on span 1, the span deflects downwards, and while there are some dynamic movements evident their amplitude is small relative to the static displacement. As the truck passes onto span 2 the midspan of span 1 deflects upwards slightly. Once the truck leaves the bridge at time, $t=4$ seconds, the deck goes into free vibration, but the amplitudes of the displacements are very small relative to the static displacement. Figure 8(c) shows the corresponding vertical velocity response at the mid-span of span 1. While the static movements are still evident in the velocity signal the dynamic movements are much clearer. Figure 8(e) shows the vertical acceleration response of the mid-span of span 1. This time the dynamic movements of the bridge dominate the signal and high amplitude localised peaks are visible in the acceleration signal as the front and rear axles enter/leave the bridge. The logarithmic decay of the acceleration signal when the bridge goes into free vibration is also evident in part (e) of the figure. The three plots on the right side of the figure (i.e., (b), (d), and (f)) show the horizontal displacement, velocity, and acceleration of the top of the pier resulting from the passage of the vehicle across 


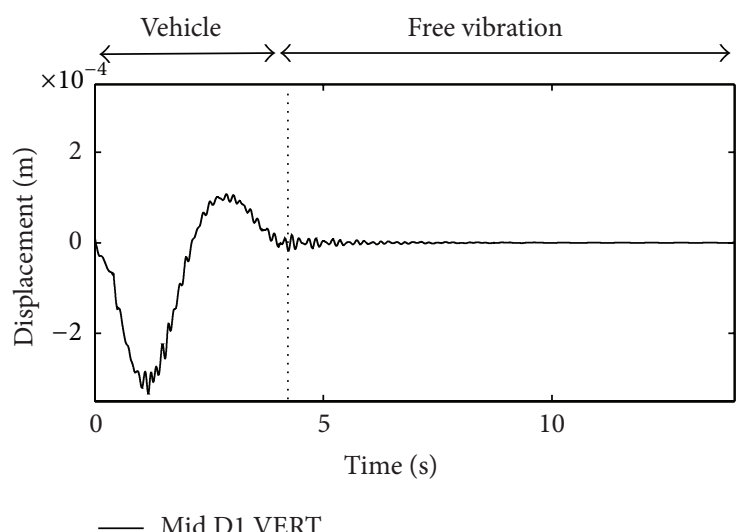

(a)

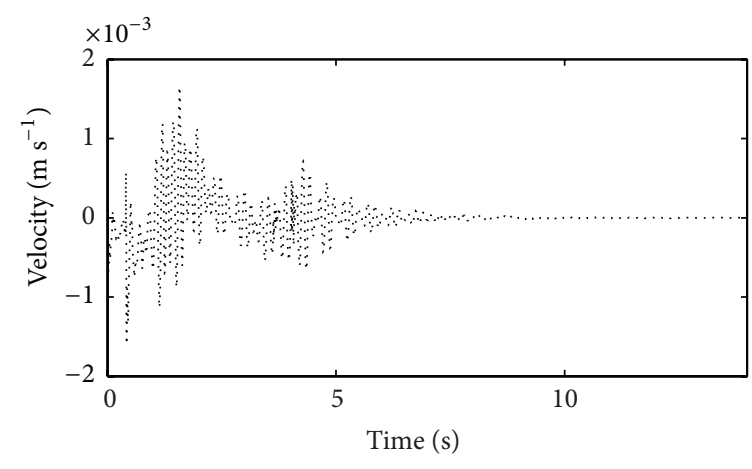

… Mid D1 VERT

(c)

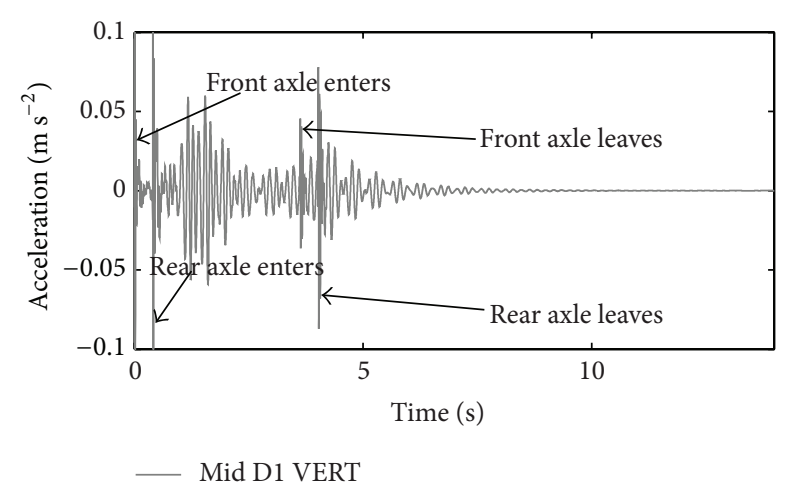

(e)

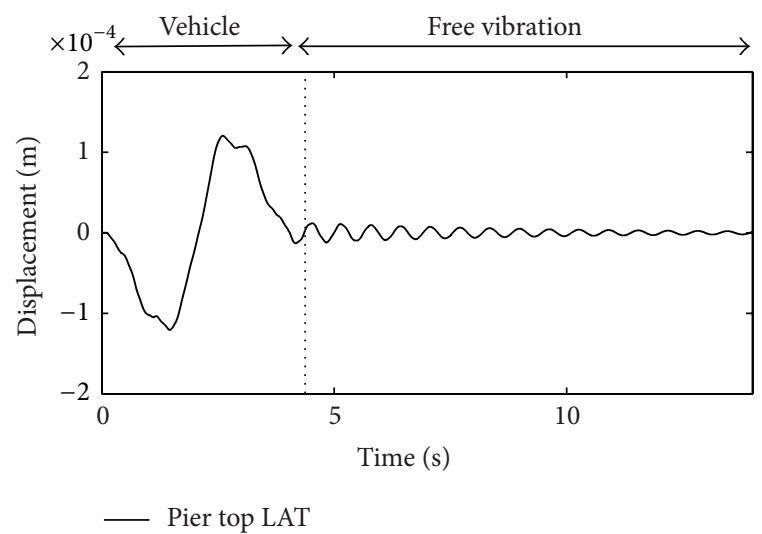

(b)

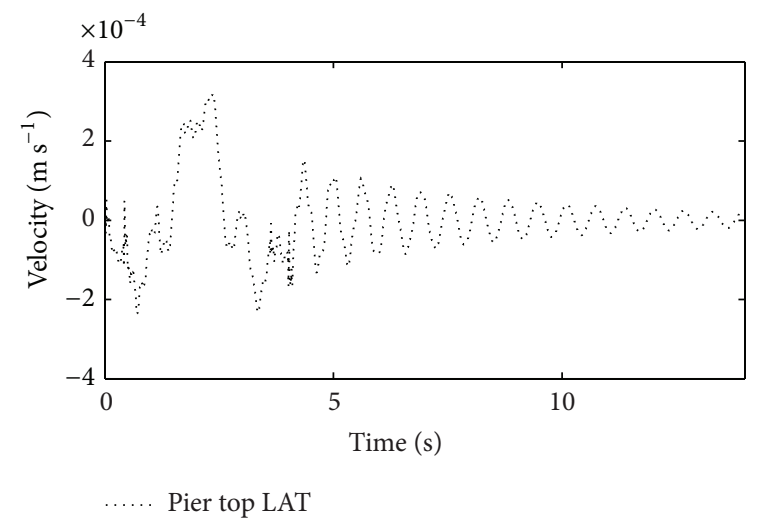

(d)

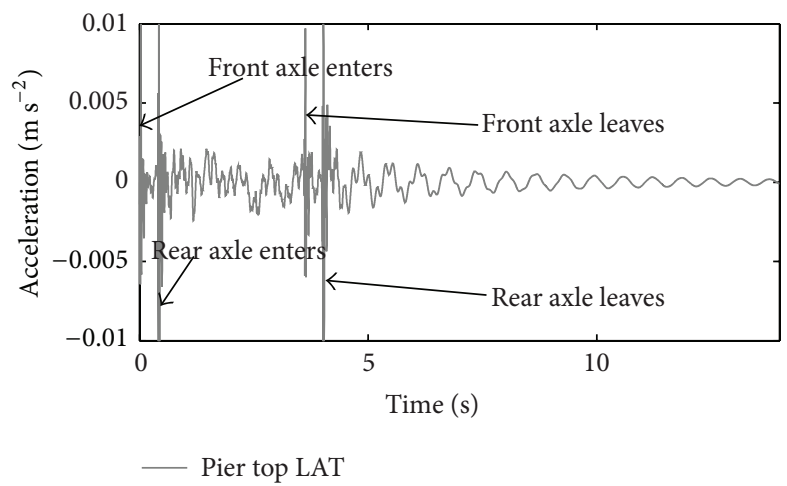

(f)

FIGURE 8: Example signals from model for vehicle traversing at $50 \mathrm{~km} \mathrm{hr}^{-1}$ with 10 seconds of free vibration and a Class " $A$ " road profile. (a) Vertical displacement response at mid-span of span 1; (b) horizontal displacement at top of pier; (c) vertical velocity response at mid-span of span 1; (d) horizontal velocity at top of pier; (e) vertical acceleration response at mid-span of span 1; (f) horizontal acceleration at top of pier. All signals are damped at $2 \%$.

the bridge. When the vehicle is on span 1 the head of the pier moves to the left (negative displacement) and when the vehicle is on span 2 the head of the pier moves to the right (positive displacement). Although there is little dynamic movement evident when the vehicle is on the bridge, once the bridge goes into free vibration the dynamic movements are clearly evident. The horizontal velocity signal shown in part (d) of the figure shows a clear dynamic component, and the frequency is noticeably lower than the frequency evident in the plot of vertical mid-span velocity (Figure 8(c)). Figure $8(\mathrm{f})$ shows the horizontal acceleration response at the top of the pier and again the peaks due to the axles entering/leaving the bridge are evident.

For the purpose of scour detection, the lateral bridge response is often the response of most interest, in that this is the response typically affected by scour in bridges of this type $[16,40]$. Therefore it is of interest to examine the parameters that affect the ability for a vehicle traversing the bridge to 


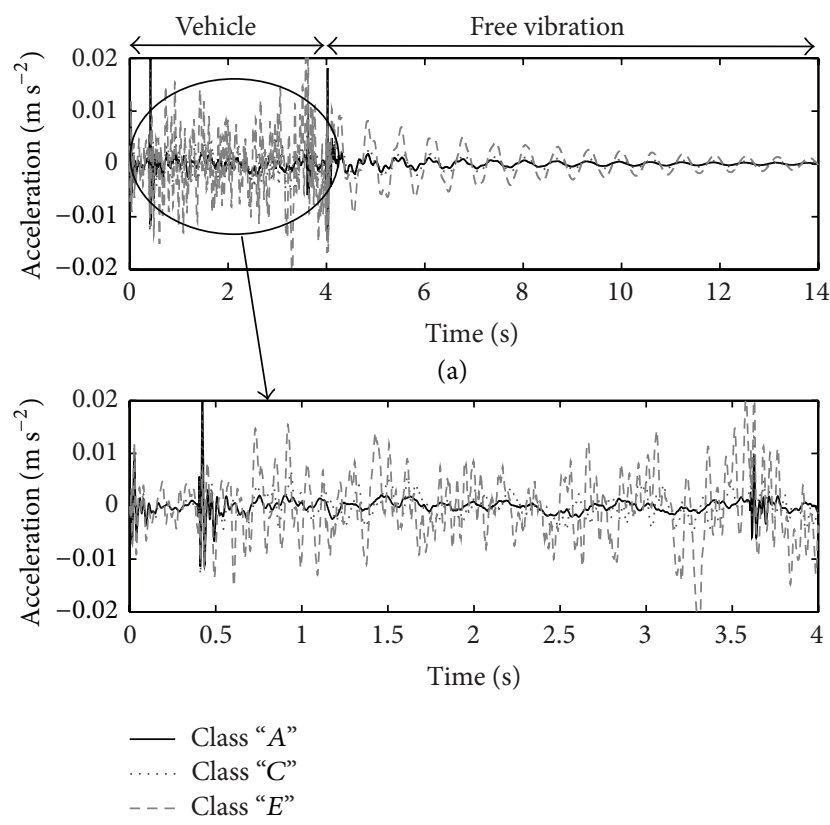

(b)

FIGURE 9: Effect of road surface roughness on lateral pier top acceleration response, (a) forced and free vibration for Classes $A$, $C$, and $E$ and (b) forced vibration only.

excite the lateral response of the structure. Figures $8(\mathrm{~b})$, $8(\mathrm{~d})$, and $8(\mathrm{e})$ show the lateral displacement, velocity, and acceleration response of the pier top due to the passage of a vehicle over a Class " $A$ " road surface. The effect of increased road surface roughness on the lateral pier top acceleration is examined in Figure 9. The vehicle traverses the bridge at $50 \mathrm{~km} \mathrm{hr}^{-1}$ and 10 seconds of free vibration (damped at 2\%) is assumed after the vehicle leaves the bridge.

Figure 9 shows the effect of increasing road surface roughness from $A$ to $C$ to $E$ on the resulting lateral acceleration response at the pier top (see Figure 6 for road profiles). The effect of increasing the surface roughness leads to higher magnitude acceleration responses for both the forced and free vibration components of the response signal. As the forced vibration component is difficult to see in Figure 9(a), part (b) shows only the forced component of the signal.

The effect of scour on the lateral acceleration response of the pier top is examined in Figure 10, whereby $5 \mathrm{~m}$ of scour is induced around the central pier foundation. For this analysis, the vehicle once again traverses the bridge at $50 \mathrm{~km} \mathrm{hr}^{-1}$ over a Class " $A$ " road surface with 10 seconds of subsequent free vibration.

Figure 10 shows the effect of $5 \mathrm{~m}$ of pier scour on the lateral acceleration response measured at the pier top due to the passing vehicle at $50 \mathrm{~km} \mathrm{hr}^{-1}$. In this figure, the period of the signal has increased with scour (see the insert showing a zoomed-in portion of the signal between 4.5 and 7 seconds). This is sensible as the effect of scour is to increase the effective length of the pier thus making it more flexible so an increased period is expected. The amplitude of the scoured signal has reduced in the free vibration. The reason for this is twofold. (i) The effect of scour reducing the confining stiffness effect

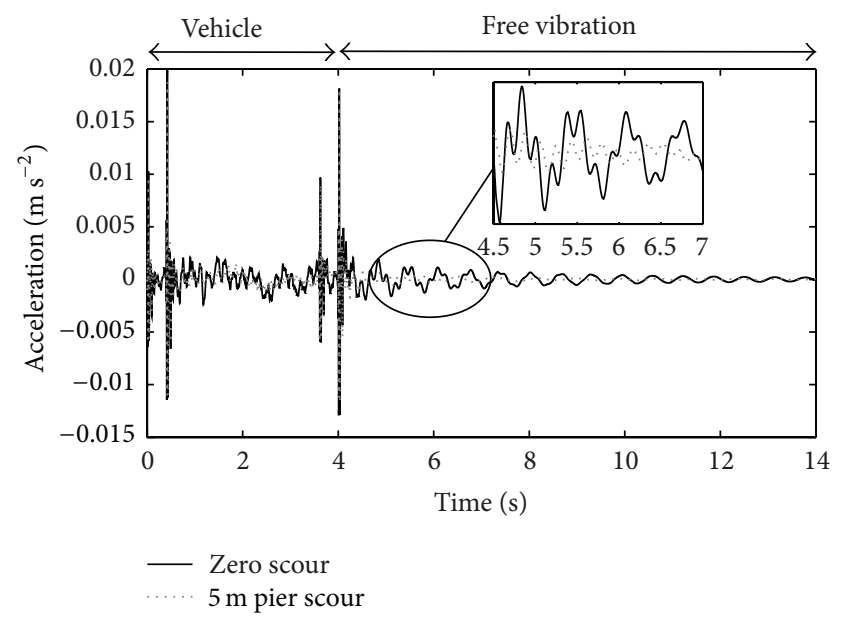

Figure 10: Difference in lateral pier top acceleration for 0 and $5 \mathrm{~m}$ scour.

of the soil leads to higher lateral translation of the pier at lower depths, meaning the pier top translation is lessened. This manifests itself as a lower pier top dynamic displacement and acceleration for increased pier scour. (ii) Interaction effects between the bridge's natural oscillation period and the velocity of the vehicle lead to differences in the free vibration amplitude depending on where the vehicle is on the bridge at a given time increment relative to the (lateral) oscillation state of the bridge itself. The location of the vehicle at a certain time means it can either increase or diminish the bridge's natural oscillatory response.

In terms of damage detection using vibrations, many SHM algorithms can be sensitive to the presence of measurement noise as well as other external disturbances. Figure 11 shows the effect of artificially adding noise to the simulated acceleration signals. For this analysis, the process outlined in (11)-(13) is used whereby noise is added by utilising the signal-to-noise ratio (SNR) as per [38]. Once again, this analysis is for a vehicle traversing at $50 \mathrm{~km} \mathrm{hr}^{-1}$, a Class " $A$ " road profile, loose sand profile, 10 seconds of free vibration, zero scour, and the lateral acceleration generated at the pier top. SNRs of 20,10, and 5 are used to show the increasing effect of noise on the time-domain acceleration signals.

Figure 11 shows the effect of increasing the noise level present in the lateral acceleration response at the top of the bridge pier. In Figure 11(a) the characteristics of the signal are easily discernible and the individual oscillations in the free vibration can be seen clearly. On the contrary, in Figure $11(\mathrm{~d})$ with a SNR $=5$, the individual oscillations are difficult to identify and it is clear that the addition of noise has significantly reduced the clarity of the signal. The addition of noise to the signals allows researchers to test the resilience of emerging SHM algorithms under increasingly challenging conditions that might be experienced in the field.

4.2. Vehicle Signals. In this section, some typical vehicle response signals are shown. The purpose of using vehicle signals as mentioned previously is to allow researchers to test indirect methods of SHM, whereby the response of the 


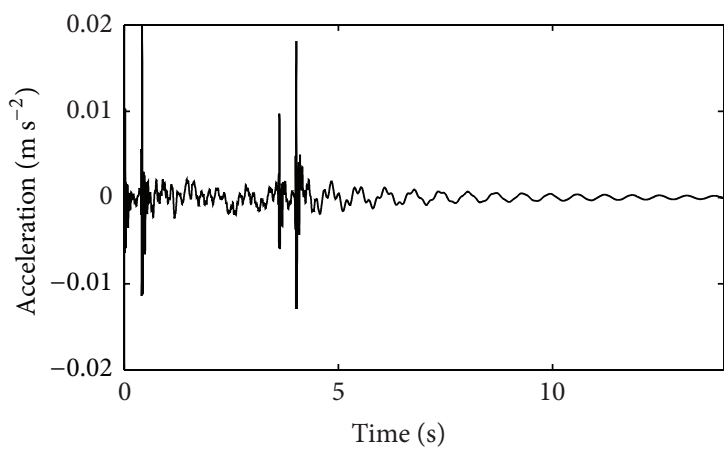

— No noise

(a)

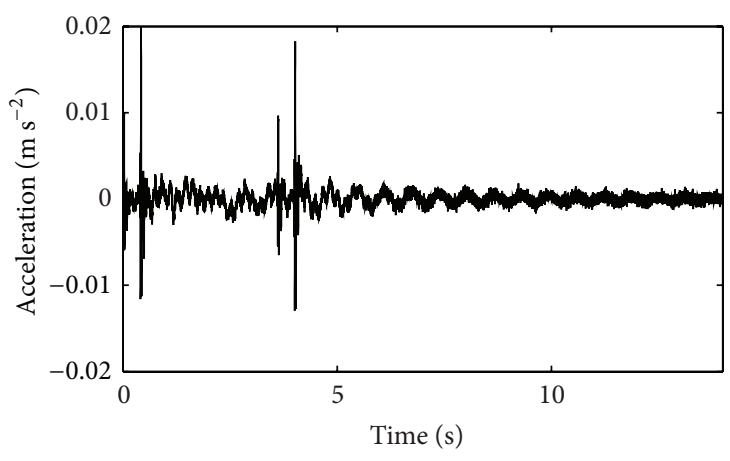

SNR $=10$

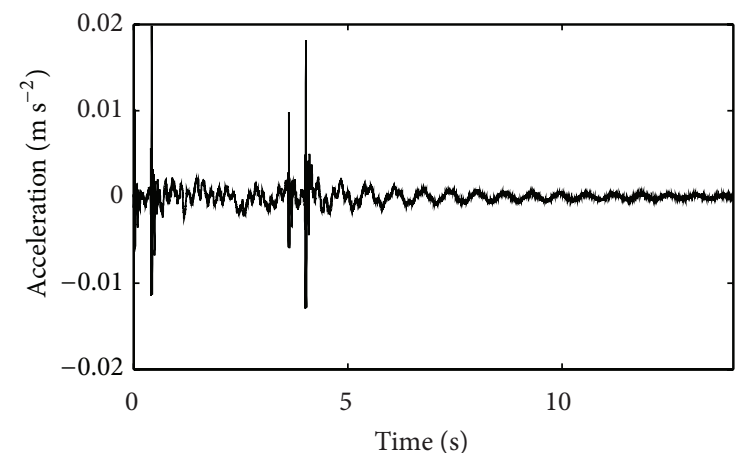

$-\mathrm{SNR}=20$

(b)

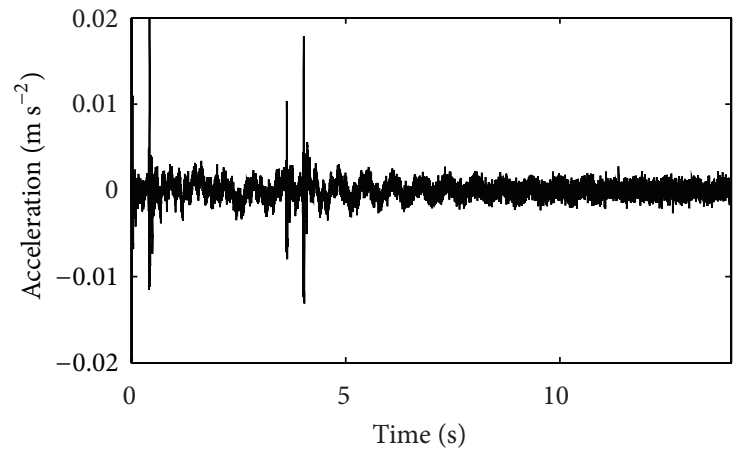

$\mathrm{SNR}=5$

(c)

(d)

FIGURE 11: Effect of noise on lateral pier acceleration response. (a) Noise-free signal; (b) signal with SNR = 20; (c) signal with SNR = 10; (d) signal with $\mathrm{SNR}=5$.

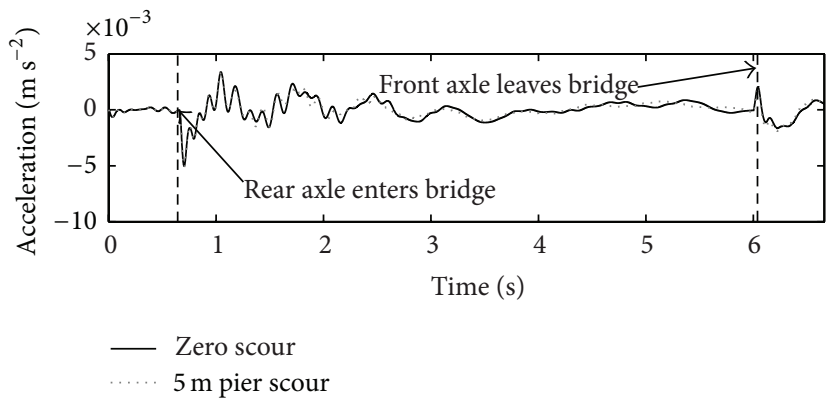

(a)

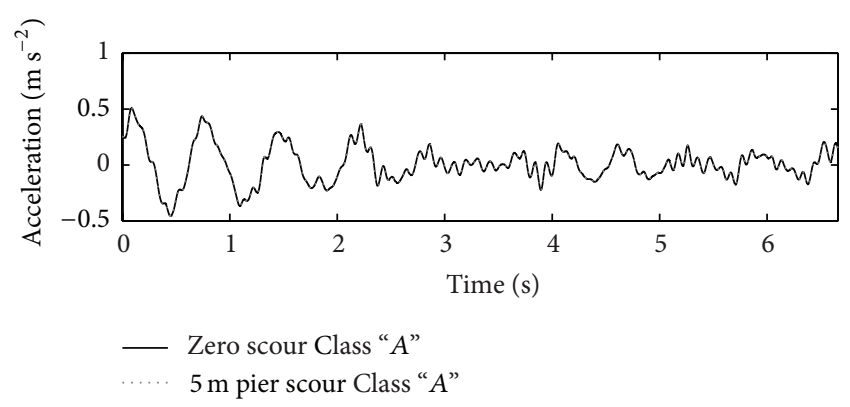

(b)

FiguRE 12: Effect of road roughness on vehicle body accelerations. (a) Vertical body acceleration over smooth road surface for zero and $5 \mathrm{~m}$ scour, (b) vertical body acceleration over Class " $A$ " road surface for zero and $5 \mathrm{~m}$ scour.

vehicle is used to infer the damage condition of the bridge. For example, a number of authors have inferred the condition of the bridge deck by analysing the vehicle response as it crossed the bridge [41, 42]. The vehicle response is sensitive to the road profile; therefore in this section the effect of road surface roughness is examined in terms of detecting the presence of scour. Figure 12 shows the vertical acceleration response of the vehicle body as it traverses a bridge at $30 \mathrm{~km} \mathrm{hr}^{-1}$ for zero and $5 \mathrm{~m}$ of pier scour. To make the simulation as realistic as possible the vehicle commences motion at an approach distance of $100 \mathrm{~m}$ from the start of the bridge; however, here only the portion of the response when the vehicle is on the bridge is shown. A loose sand profile is adopted in the bridge model and the vehicle traverses a smooth road surface in Figure 12(a) and a Class " $A$ " road surface in Figure 12(b) (see Figure 6 for road profile).

Figure 12 shows the effect of the road surface roughness on the clarity of the vertical acceleration response detected on the vehicle body as it traverses the bridge at $30 \mathrm{~km} \mathrm{hr}^{-1}$. Figure 12(a) shows the difference in vehicle body vertical 
acceleration for zero and $5 \mathrm{~m}$ pier scour as it traverses a smooth road surface. Figure 12(b) shows that the difference between zero scour and $5 \mathrm{~m}$ pier scour vertical vehicle body accelerations is difficult to observe in the presence of a Class " $A$ " road surface. The presence of a road profile may cause difficulty with some SHM schemes aiming to use vehicle measurements to detect damage/scour.

\section{Conclusion}

In this paper, the development of a novel Vehicle-Bridge-Soil Dynamic Interaction (VBSDI) model for modelling the effect of scour around integral bridge foundations is presented. The model is capable of rapidly modelling the scour response of integral bridges and incorporates realistic (validated) representations of the foundation soil stiffness as well as a realistic vehicle loading model. This paper discusses the mathematical theory and assumptions underlying the model. The model is capable of outputting dynamic displacements, velocities, and accelerations from any point on the bridge as well as on the vehicle with a view to allowing researchers to test emerging structural health monitoring techniques. Signals from the bridge can be used to test direct methods of health monitoring, whereby sensors are placed on the actual bridge structure. Signals measured on the vehicle as it traverses the bridge can also be used to test indirect methods of health monitoring, which rely on the bridge's damage feature being detected in the vehicle response. The model can vary the soil conditions from loose sand to dense sand and vary the road surface roughness from Class " $A$ " (very good) to Class " $E$ " (very poor). The effects of measurement noise can also be incorporated using two separate methods to afford a more rigorous assessment of a particular SHM technique aiming to use real (noisy) signals. Finally, some typical response data from both a bridge and a two-axle vehicle are presented by way of a case study.

The model developed in this paper is intended to aid researchers working in the area of scour detection using vibration-based methods to test emerging algorithms that they may have in development. While every effort has been made to ensure the signals from the model are as realistic as possible, it is recommended where possible to test developed techniques on actual structures. The current model aims to serve as a preliminary tool for researchers to test their techniques under a range of modelling scenarios prior to field deployment.

\section{Conflict of Interests}

The authors declare that there is no conflict of interests regarding the publication of this paper.

\section{Acknowledgments}

The authors would like to acknowledge the support of the Earth and Natural Sciences (ENS) Doctoral Studies Programme, funded by the Higher Education Authority (HEA) through the Programme for Research at Third Level Institutions, Cycle 5 (PRTLI-5), cofunded by the European
Regional Development Fund (ERDF), the European Union Framework 7 Project SMART RAIL (Project no. 285683), and the University College Dublin (UCD) Earth Institute.

\section{References}

[1] C. R. Farrar and K. Worden, "An introduction to structural health monitoring," Philosophical Transactions. Series A, Mathematical, Physical, and Engineering Sciences, vol. 365, no. 1851, pp. 303-315, 2007.

[2] A. D. Dimarogonas, "Vibration of cracked structures: a state of the art review," Engineering Fracture Mechanics, vol. 55, no. 5, pp. 831-857, 1996.

[3] E. A. Johnson, H. F. Lam, L. S. Katafygiotis, and J. L. Beck, "Phase I IASC-ASCE structural health monitoring benchmark problem using simulated data," Journal of Engineering Mechanics, vol. 130, no. 1, pp. 3-15, 2004.

[4] S. Dyke, D. Bernal, J. Beck, and C. Ventura, "Experimental phase II of the structural health monitoring benchmark problem," in Proceedings of the 16th ASCE Engineering Mechanics Conference, Seattle, Wash, USA, July 2003, http://authors.library.caltech .edu/34226/1/Report_bldg_shm_exp2.pdf.

[5] S. Foti and D. Sabia, "Influence of foundation scour on the dynamic response of an existing bridge," Journal of Bridge Engineering, vol. 16, no. 2, pp. 295-304, 2011.

[6] W. Ostachowicz and M. Krawczak, "On modelling of structural stiffness loss due to damage," in Proceedings of the 4th International Conference Damage Assessment of Structures (DAMAS '01), Cardiff, UK, 2001.

[7] M. I. Friswell and J. E. T. Penny, "Crack modeling for structural health monitoring," Structural Health Monitoring, vol. 1, no. 2, pp. 139-148, 2002.

[8] C. P. Ratcliffe, "Damage detection using a modified laplacian operator on mode shape data," Journal of Sound and Vibration, vol. 204, no. 3, pp. 505-517, 1997.

[9] M. A. Mahmoud, "Effect of cracks on the dynamic response of a simple beam subject to a moving load," Proceedings of the Institution of Mechanical Engineers, Part F: Journal of Rail and Rapid Transit, vol. 215, no. 3, pp. 207-215, 2001.

[10] X. Q. Zhu and S. S. Law, "Wavelet-based crack identification of bridge beam from operational deflection time history," International Journal of Solids and Structures, vol. 43, no. 7-8, pp. 2299-2317, 2006.

[11] D. Hester and A. González, "A wavelet-based damage detection algorithm based on bridge acceleration response to a vehicle," Mechanical Systems and Signal Processing, vol. 28, pp. 145-166, 2012.

[12] A. González, E. J. Obrien, and P. J. McGetrick, "Identification of damping in a bridge using a moving instrumented vehicle," Journal of Sound and Vibration, vol. 331, no. 18, pp. 4115-4131, 2012.

[13] L. J. Prendergast and K. Gavin, "A review of bridge scour monitoring techniques," Journal of Rock Mechanics and Geotechnical Engineering, vol. 6, no. 2, pp. 138-149, 2014.

[14] S. H. Ju, "Determination of scoured bridge natural frequencies with soil-structure interaction," Soil Dynamics and Earthquake Engineering, vol. 55, pp. 247-254, 2013.

[15] C.-C. Chen, W.-H. Wu, F. Shih, and S.-W. Wang, "Scour evaluation for foundation of a cable-stayed bridge based on ambient vibration measurements of superstructure," NDT \& $E$ International, vol. 66, pp. 16-27, 2014. 
[16] J. V. Klinga and A. Alipour, "Assessment of structural integrity of bridges under extreme scour conditions," Engineering Structures, vol. 82, pp. 55-71, 2015.

[17] L. J. Prendergast, D. Hester, K. Gavin, and J. J. O’Sullivan, “An investigation of the changes in the natural frequency of a pile affected by scour," Journal of Sound and Vibration, vol. 332, no. 25, pp. 6685-6702, 2013.

[18] S. Donohue, M. Long, K. Gavin, and P. O'Connor, "Shear wave stiffness of Irish glacial till," in Proceedings of the 2nd International Conference on Site Characterization, pp. 459-466, Porto, Portugal, 2004.

[19] L. J. Prendergast, K. Gavin, and P. Doherty, "An investigation into the effect of scour on the natural frequency of an offshore wind turbine," Ocean Engineering, vol. 101, pp. 1-11, 2015.

[20] S. M. Springman, A. R. M. Norrish, and C. W. W. Ng, "Cyclic loading of sand behind integral bridge abutments," TRL Report 146, Transport Research Laboratory, Wokingham, UK, 1996.

[21] Steel Construction Institute, "Integral bridges," 2015, http:// www.steelconstruction.info/Integral_bridges\#References.

[22] Y. W. Kwon and H. Bang, The Finite Element Method using MATLAB, CRC Mechanical Engineering Series, CRC Press, Boca Raton, Fla, USA, 2nd edition, 2000.

[23] Y. Yang, J. Yau, and Y. Wu, Vehicle-Bridge Interaction Dynamics, World Scientific, 2004, http://www.worldscientific.com/doi/ pdf/10.1142/9789812567178_fmatter.

[24] J. W. Tedesco, W. G. McDougal, and C. Allen Ross, Structural Dynamics: Theory and Applications, Addison Wesley Longman, 1999.

[25] E. Winkler, Theory of Elasticity and Strength, Dominicus, Prague, Czech Republic, 1867.

[26] S. C. Dutta and R. Roy, "A critical review on idealization and modeling for interaction among soil-foundation-structure system," Computers and Structures, vol. 80, no. 20-21, pp. 15791594, 2002.

[27] T. Lunne and H. P. Christoffersen, "Interpretation of cone penetrometer data for offshore sands," in Proceedings of the Offshore Technology Conference, Houston, Tex, USA, 1983.

[28] API, "Recommended practice for planning, designing and constructing offshore platforms-working stress design," API RP2A, American Petroleum Institute, Washington, DC, USA, 2007.

[29] Fugro, "Guide for estimating soil type and characteristics using cone penetration testing," Cone Penetration Tests, 2011, http://www.fes.co.uk/downloads/CPT-general.pdf.

[30] R. J. Jardine, F. C. Chow, R. F. Overy, and J. Standing, ICP Design Methods for Driven Piles in Sands and Clays, Thomas Telford Publishing, London, UK, 2005.

[31] G. Baldi, R. Belottini, V. N. Ghionna, N. I. Jamiokowski, and D. C. F. Lo Presti, "Modulus of sands from CPTs and DMTs," in Proceedings of the 12th International Conference on Soil Mechanics and Foundation Engineering (ICSMFE '89), vol. 1, pp. 165-170, Rio de Janeiro, Brazil, August 1989.

[32] S. A. Ashford and T. Juirnarongrit, "Evaluation of pile diameter effect on initial modulus of subgrade reaction," Journal of Geotechnical and Geoenvironmental Engineering, vol. 129, no. 3, pp. 234-242, 2003.

[33] M. F. Green and D. Cebon, "Dynamic interaction between heavy vehicles and highway bridges," Computers and Structures, vol. 62, no. 2, pp. 253-264, 1997.

[34] F. Yang and G. Fonder, "An iterative solution method for dynamic response of bridge-vehicles systems," Earthquake
Engineering \& Structural Dynamics, vol. 25, no. 2, pp. 195-215, 1996.

[35] N. K. Harris, E. J. OBrien, and A. González, "Reduction of bridge dynamic amplification through adjustment of vehicle suspension damping," Journal of Sound and Vibration, vol. 302, no. 3, pp. 471-485, 2007.

[36] D. Cebon, Handbook of Vehicle-Road Interaction, Swets \& Zeitlinger, Lisse, The Netherlands, 1999.

[37] L. Fryba, Vibration of Solids and Structures Under Moving Loads, Thomas Telford, London, UK, 1999.

[38] R. Lyons, Understanding Digital Signal Processing, Prentice Hall, Boston, Mass, USA, 3rd edition, 2011.

[39] Concast, Civil Engineering Solutions, Civil Engineering Solutions, 2014, http://www.concastprecast.co.uk/images/uploads/ brochures/Concast_Civil.pdf.

[40] A. Elsaid, Vibration based damage detection of scour in coastal bridges [Ph.D. thesis], North Carolina State University, Raleigh, NC, USA, 2012.

[41] J. Keenahan, E. J. OBrien, P. J. McGetrick, and A. Gonzalez, "The use of a dynamic truck-trailer drive-by system to monitor bridge damping," Structural Health Monitoring, vol. 13, no. 2, pp. 143-157, 2014.

[42] A. Malekjafarian and E. J. OBrien, "Identification of bridge mode shapes using short time frequency domain decomposition of the responses measured in a passing vehicle," Engineering Structures, vol. 81, pp. 386-397, 2014. 


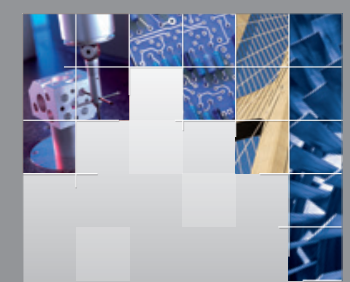

\section{Enfincering}
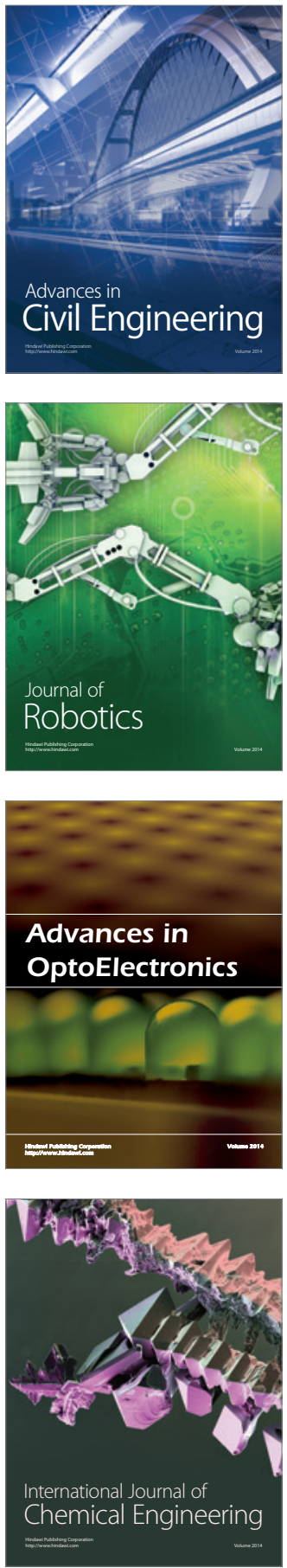

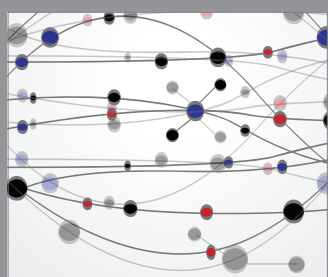

The Scientific World Journal

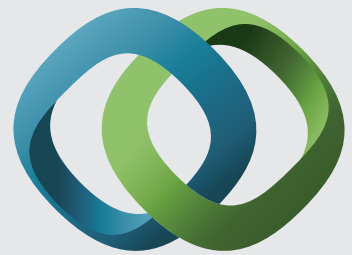

\section{Hindawi}

Submit your manuscripts at

http://www.hindawi.com
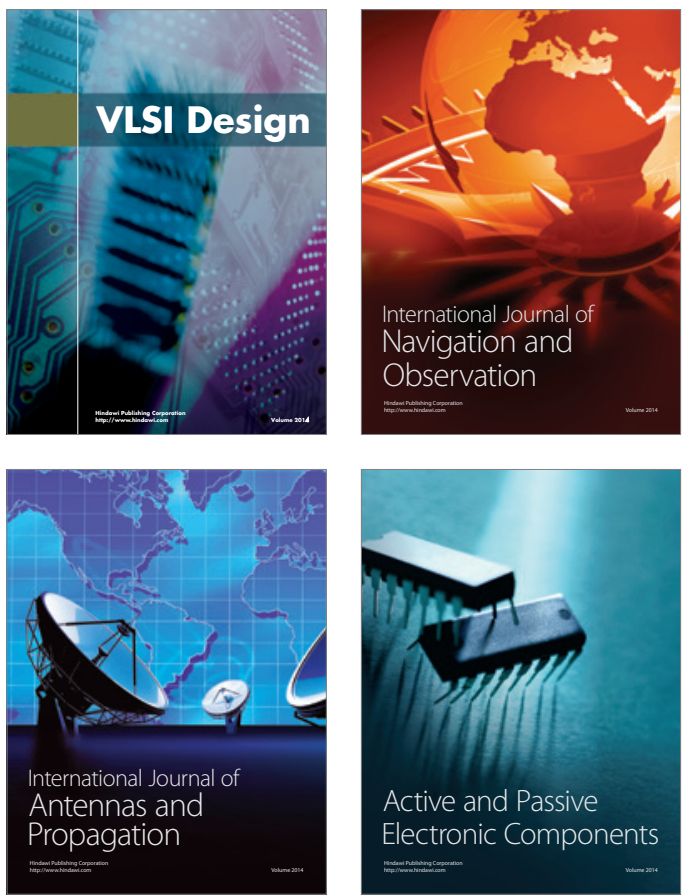
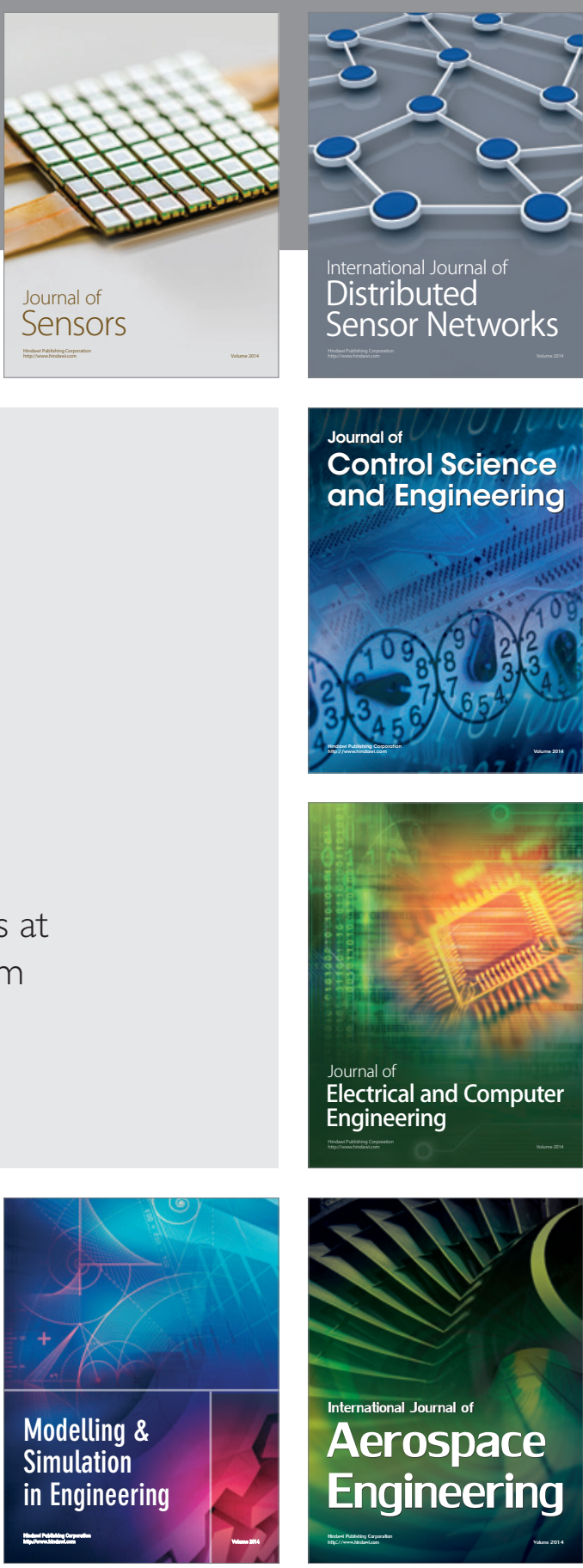

International Journal of

Distributed

Sensor Networks

Journal of

Control Science

and Engineering
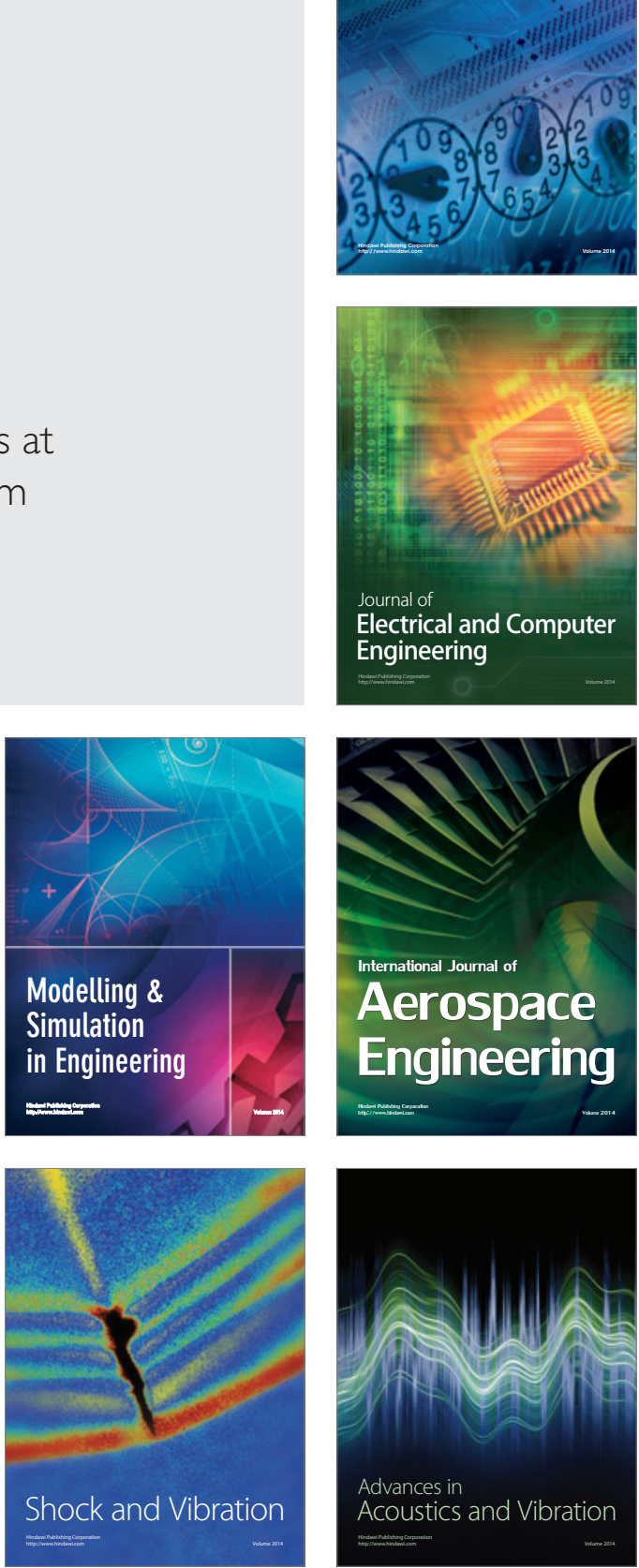